\title{
Copenhagen Quantum Mechanics Emerges from a Deterministic Schrödinger Theory in 11 Dimensional Spacetime Including Weak Field Gravitation
}

\author{
G. Doyen ${ }^{1}$ - D. Drakova ${ }^{2}$
}

Received: 4 August 2014 / Accepted: 7 May 2015 / Published online: 6 June 2015

(C) The Author(s) 2015. This article is published with open access at Springerlink.com

\begin{abstract}
We construct a world model consisting of a matter field living in 4 dimensional spacetime and a gravitational field living in 11 dimensional spacetime. The seven hidden dimensions are compactified within a radius estimated by reproducing the particle-wave characteristics of diffraction experiments. In the presence of matter fields the gravitational field develops localized modes with elementary excitations called gravonons which are induced by the sources (massive particles). The final world model treated here contains only gravonons and a scalar matter field. The gravonons are localized in the environment of the massive particles which generate them. The solution of the Schrödinger equation for the world model yields matter fields which are localized in the 4 dimensional subspace. The localization has the following properties: (i) There is a chooser mechanism for the selection of the localization site. (ii) The chooser selects one site on the basis of minor energy differences and differences in the gravonon structure between the sites, which at present cannot be controlled experimentally and therefore let the choice appear statistical. (iii) The changes from one localization site to a neighbouring one take place in a telegraph-signal like manner. (iv) The times at which telegraph like jumps occur depend on subtleties of the gravonon structure which at present cannot be controlled experimentally and therefore let the telegraph-like jumps appear statistical. (v) The fact that the dynamical law acts in the configuration space of fields living in 11 dimensional spacetime lets the events observed in 4 dimensional spacetime appear non-local. In this way the phenomenol-
\end{abstract}

\footnotetext{
$凶 \quad$ D. Drakova drakova@inorg.chem.uni-sofia.bg; nhdd@chem.uni-sofia.bg

G. Doyen gerold@gerold-doyen.de

1 Ludwig-Maximilians Universität, Munich, Germany

2 Faculty of Chemistry, University of Sofia, Sofia, Bulgaria
} 
ogy of CQM is obtained without the need of introducing the process of collapse and a probabilistic interpretation of the wave function. Operators defining observables need not be introduced. All experimental findings are explained in a deterministic way as a consequence of the time development of the wave function in configuration space according to Schrödinger's equation without the need of introducing a probabilistic interpretation.

Keywords Emerging quantum mechanics - Entanglement to gravitons - Weak field gravity $\cdot$ Chooser $\cdot$ Particle localization $\cdot$ Wave-particle duality

\section{Introduction}

The problem of whether quantum mechanics, including the postulated concept of random collapses, provides a real representation of the world or whether it implies just a probabilistic interpretation of experimental data is still discussed controversially. Whereas Penrose suggests that collapse is a dynamics arising in a natural way from gravity [1-6], Omnes claims that decoherence solves the problem and, hence, objectification in quantum mechanics is a non-existent problem [7]. On the other hand Bassi and Ghirardi point out the internal contradictions of the decoherence program [8]. Pearle also refers to the conceptual difficulties of the decoherence theory [9]. A suggestion to generalize relativistically the dynamical collapse models has been presented by Bedingham et al. [10] and by Tumulka [11,12]. Kiefer and Joos maintain that the dynamical collapse models invoking gravity may be simply drowned by environmental decoherence and hence are to be considered as "excess baggage" [13-15]. As far as locality is concerned, d'Espagnat contends that decoherence theory does not reconcile physics with the objective existence of phenomena (strong realism) $[16,17]$. Dürr and Teufel maintain that decoherence does not create the facts of our world, but rather produces a sequence of fapp-redundancies, which physically increase or stabilize decoherence. They believe that physical theory should describe the behaviour of real objects, located in physical space [18]. In the present paper we report a theory which is in the spirit of quantum realism.

As is well known and well accepted, the Schrödinger equation evolves a state, which qualifies as representing real world, into a state which is a superposition of real world states and as such does not qualify as a real world state. To handle this problem von Neumann [19] introduced a second dynamics which is not unitary and serves as reduction on real world states. This second dynamics is referred to as collapse or state vector reduction and cannot presently be described by a mathematical theory. We refer to this state of quantum theory as Copenhagen quantum mechanics (CQM).

In recent years there have been attempts to construct theories where CQM and/or quantum field theory emerge in the low energy (infrared) limit of a (higherdimensional) theory which is capable of combining quantum mechanics and general relativity. Superstring theory and/or M-theory [20,21] are the most well-known examples. According to a different theory developed by 't Hooft the fundamental laws valid at the Planck scale are derived from Newton's law, but are different. In this theory CQM emerges as the low energy limit due to a chaotic (dissipative) dynamics (loss 
of information) with a set of attractors operating during the development of the system from the Planck scale to the microscopic (atomic) scale. Classical variables can assume any arbitrary value out of a continuum while quantum states are discrete [2225]. Inspired by 't Hooft's idea, Blasone et al. [26] show that the combined evolution of two classical, dissipative harmonic oscillators results in a linear harmonic quantum oscillator with a zero point energy.

Adler proposes that CQM is an emergent phenomenon arising from a deeper level of dynamics via an extension of classical dynamics to non-commuting matrix variables, with cyclic permutation inside a trace used as the basic calculational tool. CQM is then shown to emerge as the statistical thermodynamics of this underlying theory, with the canonical commutation-anticommutation relations derived from a generalized equipartition theorem. State vector reduction and probabilistic interpretation of CQM are then argued to follow from Brownian motion like corrections to the Schrödinger equation $[27,28]$. Smolin considers classical matrix models with an explicit stochastic noise giving rise to quantum behaviour [29].

Biró et al. [30] demonstrate that a classical (Euclidean) field theory living in five dimensions can behave in the infrared limit like a quantum system, if it is only observed in four dimensions. Hadley argues that the fundamental logic of CQM results from general relativity. In his theory elementary particles are not separate objects living in 4 dimensional spacetime, but rather constituents (local deformations) of spacetime. General relativity contains past and future in a deterministic manner. The statistical character of CQM is then a consequence of the missing knowledge of the future [31].

A different approach is to modify the Schrödinger equation in such a way that the collapse dynamics is included in its solution. These are the so called collapse models $[8,9,32]$. For a detailed review see refs. [8,9].

It is often stated that CQM explains all known phenomena and experimental findings and that experimental hints to a different physics are not available [33]. This is clearly not true. With the advent of experimental techniques, which provide data with time resolution and spacial resolution on the atomic scale of the dynamics of chemical and physical processes on solid surfaces in a non-destructive way, CQM is challenged. Within CQM collapse of the total wave function might be postulated as the process leading to the localization of adsorbed atoms and molecules on solid surfaces, as they are imaged in experiments with the low-temperature scanning tunnelling microscope $[34,35]$. The two-dimensional periodicity of the solid surfaces means delocalization of the wave function in two dimensional Bloch waves resulting from the time development of the wave packets according to Schrödinger's equation. It would imply that an initially localized adparticle should after some time be observed anywhere on equivalent sites on the surface with equal probability. This is, however, never observed. An extension of CQM by including the system and the environment is often considered under the heading of decoherence theory. According to this theory localization via interaction, "permanent measurement" by environmental particles (phonons, tomonagons, photons, plasmons, etc.) is suggested. But it cannot be the explanation either. This is so because at low temperatures the available environmental excitations have very long wavelengths compared to the size of the adsorbed particles. Furthermore, when adsorbed particles jump between two adsorption sites, the movement occurs as telegraph-signal like jumps and not as the smooth Rabi oscillations predicted by 
Schrödinger's equation [36]. The diffusion rates in the quantum diffusion regime of the same adsorbate on similar metal surfaces, measured in different experiments, differ by several orders of magnitude $[35,37]$. CQM, with the coupled dynamics of the adsorbate motion and the substrate phonons taken into account, provides results, which drastically differ by nearly nine orders of magnitude from the experimental diffusion rates [38]. The desorption reaction of CO, induced by tunnelling electrons in the scanning tunnelling microscope [39], can be explained only by assuming that the tunnelling electron is transiently localized in the close vicinity of the adsorbate [40]. The survival of metastable atoms in interaction with adsorbate covered solid surfaces [41,42] presents a further problem, which cannot be resolved within CQM. The survival probability of metastable atoms $\mathrm{He}^{*}$ on a metal surface, covered with $\mathrm{CO}$, is attenuated compared to the deexcitation probability in a scattering experiment between a beam of $\mathrm{He}^{*}$ and $\mathrm{CO}$ in the gas phase, which is unexpected and not explained within CQM upto now. In Ref. [43] an understanding has been suggested, based on the different dimensionality of the scattering continua in the gas phase and on the solid surface. This has been termed the dimensionality effect [44] and is in the spirit of the theory developed here.

Recently, a programmable quantum annealing machine has been built which uses quantum effects to minimize a cost function [45]. The question has been raised whether the behaviour of this D-Wave computer [46] can be described by classical statistical mechanics, an approach called simulated annealing, or whether CQM has to be invoked in order to explain the operation of D-Wave. It has been established experimentally by Chiorescu et al. [47] that a single flux qubit loses coherence within nanoseconds, i.e. within a few nanoseconds the behaviour of a single flux qubit can no longer be described by CQM. In Ramsey interferometry experiment a $\frac{\pi}{2}$ microwave pulse initializes oscillations between the macroscopic supercurrents in the flux qubit in two reverse directions, which are interpreted as oscillating probability for occupation of each current state. The envelope of the oscillations of this probability decays, providing a "coherence time" of one current state of the order of $20 \mathrm{~ns}$. These observations are interpreted to demonstrate that within nanoseconds the behaviour of a single flux qubit can no longer be described by CQM.

On the other hand, Johnson et al. show that in a macroscopic system of an eightflux-qubit chain the quantum annealing of the system at low temperature results in quantum tunnelling in the global energy minimum, with a temperature independent freezing time [48]. Furthermore the experiment of Dickson et al. [49] using 16 flux qubits of a superconducting quantum processor and quantum annealing with the help of applied transverse magnetic fields shows that, even with annealing time eight orders of magnitude longer than the time, determined for the decay of the oscillations described above, the system behaves as predicted by CQM. Classical models do not reproduce the experimental results.

We applied the theory presented in this contribution successfully to explain why does the D-Wave computer operate as a quantum computer over minutes, despite that the effects of the thermal environment are on a timescale of nanoseconds. An understanding is provided in the framework of the present theory [50].

It appears that the localization of quantum particles via entanglement to environmental continua of high density of states is a necessary condition for various reactions 
on solid surfaces. Particle localization in a collapse process, as suggested in CQM is, however, not the result of the solution of Schrödinger's equation or of any well defined dynamics. The examples from the previous paragraph illustrate that on a nanoscale level on solid surfaces there are dynamical processes which cannot be accounted for by CQM. In all cases mentioned, entanglement with an environment obviously plays a role. However, the environmental excitations usually discussed in the context of these low temperature experiments, for instance phonons, tomonagons, plasmons etc., are not capable to localize atoms or electrons on a solid surface because of their long wavelength. A new explanation is needed.

We develop a theory where CQM emerges from a quantum field theory, which treats gravitation in eleven spacetime dimensions in the weak field limit. Entanglement to soft modes in the hidden spacial dimensions leads to particle localization, which is a necessary condition for the processes on the atomic scale, listed in a previous paragraph. Changes of localization sites occur as a telegraph like dynamics with entanglement to low frequency modes and in the limit of vanishing interaction strength. Non-locality in four spacetime dimensions is implicit in the theory. It also is capable of describing and explaining the experiments on solid surfaces mentioned beforehand, which was found impossible within CQM.

There exist many attempts to account for the effects of gravity on a quantum system by modifying Schrödinger's equation including stochastic attractive Newtonian gravitational fields in 4 dimensional spacetime [51-57] or introducing a nonlinear "Schrödinger-Newton" equation $[1,51,52]$. The interpretation is based on the tendency of the off-diagonal elements of the reduced density matrix of a quantum system in interaction with the external gravitational field to attenuate, and is assumed to imply transition to classical and random behaviour. Instead, gravitation has been suggested as the origin of the noise field in collapse models [9]. Penrose's intensive attempts also suggest gravitation as the origin of collapse [1-6]. With the purpose to describe localization and transition of the quantum mechanical superposition of states to classical states, even the decay mechanism is often postulated [1,51,52]. Within the framework of continuous spontaneous reduction models [32] Pearle and Squires interpret the classical scalar field, which causes collapse, as the gravitational curvature scalar, however, gravity is treated semiclassically [58,59]. In the work of Anastopoulos the gravitational field is considered as leading to classical behaviour, however, not for microscopic particles [60]. Spacetime fluctuations due to stochastic backgrounds of gravitational waves [61] or metric fluctuations [62] have also been suggested to lead to unavoidable transition of quantum particles to classical behaviour.

The aspects of CQM are shown in this paper to emerge from entanglement due to an extremely localized and weak interaction between matter fields and a massless boson field of high mode density. The exact form of the Lagrangian for the interacting fields will be developed in Sect. 3-5. In Sect. 2 we investigate the structure of a theory based on an extremely local and weak interaction with a high density continuum in 11 dimensional spacetime. This includes compactification of the hidden dimensions (Sect.2.1.1), determination of the density of states (Sect.2.1.2) and the special kind of solution not obtainable in perturbation theory (Sects. 2.2, 2.2.1). The properties of the solution are exemplified for the case of diffraction from nano lattices. In Sects. 6 
and 7 the ontology of our Emerging quantum mechanics (EQM) is set in relation with that of Copenhagen and Bohmian quantum mechanics.

\section{The Structure of a Deterministic Schrödinger Theory in 11 Dimensional Spacetime Including Weak Field Gravitation}

As mentioned in the introduction a possible theory for the unification of general relativity and quantum mechanics is superstring theory and/or M-theory [20,21]. The equations of superstring theory are mathematically consistent only, if the strings move in 10 dimensional space. According to M-theory spacetime can besides onedimensional strings also contain branes of various dimensions. Strings can be restricted to subspaces of spacetime, if they are bound to branes. The physical laws depend on the geometry and dimensions of the branes and the hidden dimensions.

\subsection{Hidden Dimensions}

First we briefly summarize the way we think about this subject. A single hidden dimension can only have the shape of a circle. More extra dimensions can have a variety of shapes (called topologies) such as spheres, tori, connected tori, etc. differing in length and diameter. This results in a gigantic number of possible geometries. In addition there are parameters describing the positions of the branes and the fluxes around the tori. Each configuration of branes and hidden dimensions has a different energy. If there are no fields in 4 dimensional spacetime this corresponds to the vacuum energy. For a given three dimensional brane the geometry of the hidden dimensions is determined by minimizing this vacuum energy. The multitude of possible geometries results in the so called landscape of string theory. Our universe has to correspond to a minimum on the landscape with a relatively small vacuum energy, the geometry of branes and hidden dimensions corresponding to our universe is, however, unknown. For the purpose of this investigation we assume the simplest possible case, namely a flat 3-dimensional brane and a seven dimensional sphere representing the hidden dimensions. All known fields except for the gravitational field are assumed to live only on the three dimensional brane. Except for the existence of such a three dimensional brane and the spherically compactified hidden dimensions, no other properties of superstring theory and/or M-theory are needed.

String theory can be quantized in various ways, one possibility being string field theory in high dimensions. This has been demonstrated by Witten, as it is described for instance on p. 105 in the book by Becker et al. [20,21], which contains the references to the original work. The simplest example is the Nambu-Goto string action in $D$ dimensions which is easily seen to be equivalent to a quantum field theory of $D$ massless scalar fields in two dimensional spacetime (see e.g. Zee [63, pp. 452, 453]). Quantum field theory is conveniently treated by the path integral method and it is explicitly proved in the book by Gross [64] that the path integral method is fully equivalent to the Schrödinger equation. New physics which might appear at the Planck scale is of no relevance in what we discuss here. 


\subsubsection{Compactification of the Hidden Dimensions}

The basic idea of how CQM emerges in our theory is that the gravitational interaction provides a chooser that guides a diffracted matter field to a particular site on the screen. Assume the simple case that a point like source emitting the matter field is exactly in the center of a three dimensional sphere of radius 1 meter. The area of the sphere is then $4 \pi \mathrm{m}^{2} \approx 16 \times 10^{20} \mathrm{bohr}^{2} \approx 2 \times 10^{21} \mathrm{bohr}^{2}$. Assuming an area of the lattice unit cell of the screen of 20 bohr $^{2}$, there will be $10^{20}$ sites on the screen where the matter field could localize ("collapse"). The idea is that the sites are fully equivalent but vary slightly in energy and geometry. A realistic estimate for this is a variation in energy of $\approx 10^{-3} \mathrm{eV}$ and a variation in geometry of $\approx 10^{-1} \mathrm{bohr}$.

The average spacing between two adjacent energy levels is $10^{-3} \mathrm{eV} / 10^{20}$ sites $=10^{-23} \mathrm{eV}$. If the only criterion is energy then the chooser has to distinguish energy levels on a scale of $10^{-23} \mathrm{eV}$ in order to select a single site. Analogously the average deviation between two similar geometries will be $10^{-1} \mathrm{bohr} / 10^{20}$ sites $=10^{-21}$ bohr. If the only criterion is geometry then the chooser has to distinguish geometry on a scale of $10^{-21}$ bohr in order to select a single site. If we assume that the chooser is sensitive to geometric differences of $10^{-5}$ bohr then $10^{16}$ sites would be available for a choice. This means then that the chooser should distinguish on an energy scale of $10^{-3} \mathrm{eV} / 10^{16}$ sites $=10^{-19} \mathrm{eV}$ in order to select a single site.

The consequence is that the interaction energy between a quantum of the matter field and a site on the screen should be of this order of magnitude. A larger interaction energy cannot lead to site selection. A significantly smaller interaction energy would mean that either very many quanta of the matter field have to be emitted from the source, before a "collapse" can be registered, or that it would take months or years before a quantum can be registered on the screen. Both cases violate strongly the experimental findings.

The required interaction strength is much weaker than any kind of electrodynamical, chemical or van der Waals interaction. This means that coupling to phonons or electron-hole pairs, i.e. "measurement by the environment", is not capable of describing the "collapse" or measurement process which is at the heart of the so called wave-particle duality. On the other hand the required interaction strength is much stronger than the gravitational interaction in 4 dimensional spacetime. Consider two protons at a distance of $r=6$ bohr and mass $M=2 \times 10^{3}$ a.u. Their gravitational interaction energy is $G M^{2} / r \approx 10^{-36} \mathrm{eV}$, where we inserted a value of $G=10^{-40}$ atomic units for Newton's gravitational constant. This is 17 orders of magnitude smaller than required. The only possibility for obtaining the required order of magnitude for the interaction energy appears to be a gravitational field in 11 dimensional spacetime with compactified hidden dimensions.

The gravitational potential provided by a pointlike mass $M$ in 4 dimensional spacetime is:

$$
V_{\text {grav }}^{(4)}(r)=-\frac{G M}{r}
$$

In 11 dimensional spacetime (10 space dimensions) the gravitational potential is: 


$$
V_{\text {grav }}^{(11)}(r)=-\frac{G^{(11)} M}{\pi^{7} r^{8}}
$$

The gravitational law Eq. (2) cannot be valid for large separations $r$ as this would violate the experimentally verified classical law Eq. (1). Therefore the hidden dimensions are rolled up (compactified) to a diameter $2 a$ so that at large distances the separation in the hidden dimensions never exceeds $2 a$. Equating the classical and the 11 dimensional gravitational law at large distances, we obtain

$$
-\frac{G M}{r}=-\frac{G^{(11)} M}{(2 a \pi)^{7} r} \rightarrow G^{(11)}=(2 a \pi)^{7} G
$$

This choice makes the two laws Eqs. (1) and (2) agree at separations larger than $2 a$. Inserting Eq. (3) in Eq. (2) yields at $r=1$ bohr:

$$
V_{\text {grav }}^{(11)}(r=1)=-G M(2 a)^{7}
$$

This means that at $r=1$ bohr the gravitational interaction is $(2 a)^{7}$ times stronger than predicted by the classical law Eq. (1).

The dependence of $G^{(11)}$ on the compactification radius is then:

$\begin{array}{lc}\text { a(bohr) } & G^{(11)} / \pi^{7} \text { (a.u.) } \\ 10^{4} & 10^{-10} \\ 10^{3} & 10^{-17} \\ 10^{2} & 10^{-24} \\ 10 & 10^{-31}\end{array}$

From the selection criterion described above we require $\frac{G^{(11)} M}{\pi^{7} r^{8}} \approx 10^{-20}$ atomic units at $r=6$ bohr and $M=2 \times 10^{3}$, hence $\frac{G^{(11)} M}{\pi^{7} 6^{8}} \approx \frac{G^{(11)} 2 \times 10^{3}}{\pi^{7} 10^{6}} \approx 2 \times 10^{-3} \frac{G^{(11)}}{\pi^{7}} \approx$ $10^{-20}$ which implies $\frac{G^{(11)}}{\pi^{7}} \approx 5 \times 10^{-18}$. For our chooser we would therefore need a compactification radius of roughly $10^{3}$ bohr. This is enormously larger than the Planck length. A chooser needs large extra dimensions. In the theory of Arkani-Hamed et al. it is also claimed that the hidden dimensions need not be compactifed on the Planck scale, they can be as large as a fraction of a millimeter or even infinite $[65,66]$.

\subsubsection{Graviton Mode Density}

The chooser can only work, if gravitons are available at an energy separation which is of the order of the gravitational interaction strength for any kind of matter field quantum. This is only possible, if the density of graviton modes is sufficiently large. The density of graviton modes has been calculated in Ref. [67]. Due to its importance the argument is repeated here. The calculation runs parallel to the evaluation of the mode density in the electromagnetic case. We expand the gravitational radiation field in the eigenmodes of resonators with fixed frequency, polarization, and field distribution. Although the selection of modes is in principle arbitrary, the most popular expansion 
uses the eigenmodes of rectangular boxes with perfectly reflecting walls. The mirrors at position 0 and $a$ impose the boundary conditions that the field vanishes at those positions. The eigenmodes have field distributions that vary with $\sin (n \pi x / a)$, where $n$ is the mode number, $x$ the spacial variable and $a$ the separation of the two mirrors. The spatiotemporal variation of the field is

$$
F_{n}=\sin (n \pi x / a) \mathrm{e}^{i \omega_{n} t}
$$

The wave number $k=(n \pi / a)$ determines the angular frequency $\omega_{n}=\frac{n \pi \mathrm{c}}{a}$. The one dimensional density of modes in $k$-space is

$$
\rho_{k}=\frac{a}{\pi}
$$

Using the dispersion relation for gravitons $\epsilon_{\kappa}=\kappa \mathrm{c}$, with $\mathrm{c}$ the velocity of light, $\kappa=\sqrt{k_{1}^{2}+k_{2}^{2}+k_{3}^{2}+k_{4}^{2}+\cdots+k_{d}^{2}}$ and $d$ the dimension of gravitational $k$-space, we write for the graviton mode density

$$
\rho_{\text {grav }}(E)=\sum_{\kappa} \delta\left(E-\epsilon_{\kappa}\right)=\int \mathrm{d}^{d} \kappa \rho_{\kappa}^{d} \delta\left(E-\epsilon_{\kappa}\right) .
$$

Transforming to spherical coordinates $\eta$ one obtains [68]:

$$
\rho_{\text {grav }}(E)=\int \mathrm{d} \eta \eta^{d-1} \delta\left(E-\epsilon_{\kappa}\right) \rho_{\kappa}^{d} \frac{\pi^{d / 2}}{\Gamma\left(1+\frac{d}{2}\right)} .
$$

Specializing to 10 spacial dimensions and substituting $\mathrm{d} \eta=\mathrm{d} \epsilon / \mathrm{c}$ one obtains

$$
\begin{aligned}
\rho_{\text {grav }}(E) & =\frac{1}{\mathrm{c}} \frac{\pi^{5}}{\Gamma(1+5)} \rho_{\kappa}^{d} \int \mathrm{d} \epsilon \frac{\epsilon^{d-1}}{\mathrm{c}^{d-1}} \delta(E-\epsilon) \\
& =\frac{E^{9}}{\mathrm{c}^{10}} \frac{\pi^{5}}{5 !}\left(\frac{L}{\pi}\right)^{3}\left(\frac{a}{\pi}\right)^{7}
\end{aligned}
$$

where $L$ is the normalization length of the macroscopic three dimensional space and $a$ is the normalization length of the hidden dimensions.

The density of states for the two dimensional movement of an adparticle of mass $M$ is $\rho_{2 D}=\frac{2 M L^{2}}{\pi}$ which is of the order of $10^{17}$ for a proton. Therefore the relationship between the mode density of gravitons at energy $E$ and the two dimensional adparticle motion is

$$
\frac{\rho_{\text {grav }}}{\rho_{2 D}}=\frac{\frac{E^{9}}{\mathrm{c}^{10}}}{2 M} \frac{\pi^{5}}{5 !} \frac{L a^{7}}{\pi^{9}} \approx 10^{34} .
$$


For the wave vector of the graviton we assumed $\kappa \approx 10 \mathrm{bohr}^{-1}$, which corresponds to a wave length of 1 bohr $(\lambda=2 \pi / \kappa \approx 1$ bohr $)$, and $L \approx 10^{7}$ bohr. With the choice $a \approx 10^{4}$ bohr and $M=2000$ a.u., the graviton energy is of order of $\epsilon_{\kappa} \approx 10^{3}$ Hartree.

In conclusion the graviton continuum appears infinitely dense compared to the continua describing any particle motion in our three dimensional space.

\subsection{Characteristics of the Solution}

In quantum field theory the standard way of solving a problem is via perturbation theory (Feynman diagrams). This approach does not work at all in our case. The reason is that the solution we are looking for appears in the limit of very small (vanishing) interaction strength (cf. Sect.2.1.1). Expanding in terms of powers of a coupling constant, which then tends to zero, yields zero in every order and for the final summation. This problem is known in quantum field theory, where topological (soliton) solutions appear for coupling constant tending to zero (cf. e.g. Zee [63] who refers to this situation as "breaking the shackles of Feynman diagrams"). In our case the entanglement between the matter field and gravitons becomes maximal in the (mathematical) limit, where the interaction strength $V_{\text {grav }}$ tends to zero while at the same time the graviton density of states $\rho_{\text {grav }}$ tends to $\infty$ so that $V_{\text {grav }} \rho_{\text {grav }}$ stays non-zero and finite.

The method we have to pursue is solving the Schrödinger equation in configuration space. In quantum field theory the wave function becomes a wave functional, i.e. it depends directly on the fields. Considering for illustrative purposes only two boson fields, the scalar matter field $\phi\left(t, x_{1}, x_{2}, x_{3}\right)$ and the gravitational field $\zeta\left(t, x_{1}, x_{2}, \ldots, x_{10}\right)$, we have to solve the Schrödinger equation

$$
\begin{aligned}
& \mathrm{i} \frac{\partial}{\partial t} \Psi\left(\phi\left(t, x_{1}, x_{2}, x_{3}\right), \zeta\left(t, x_{1}, x_{2}, \ldots, x_{10}\right)\right) \\
& \quad=\mathrm{H} \Psi\left(\phi\left(t, x_{1}, x_{2}, x_{3}\right), \zeta\left(t, x_{1}, x_{2}, \ldots, x_{10}\right)\right) .
\end{aligned}
$$

Reasonable solutions can be obtained by adapting techniques, which have been developed in theoretical quantum chemistry under the heading of "configuration interaction" (CI).

Consider the set of fields $\left\{\phi^{n} \zeta^{m}\right\}$ forming a Hilbert space. Expanding the fields in $\operatorname{modes} \phi_{\alpha}$ and $\zeta_{\beta}$ :

$$
\begin{gathered}
\phi=\sum_{\alpha=1}^{\infty} C_{\alpha} \phi_{\alpha} \\
\zeta=\sum_{\beta=1}^{\infty} D_{\beta} \zeta_{\beta}
\end{gathered}
$$

we write the wave functional in Dirac notation as

$$
|\Psi\rangle=\sum_{\left\{n_{\alpha}\right\},\left\{n_{\beta}\right\}} A_{\left\{n_{\alpha}\right\}\left\{n_{\beta}\right\}} \otimes_{\alpha=1}^{\infty}\left|n_{\alpha}\right\rangle \otimes_{\beta=1}^{\infty}\left|n_{\beta}\right\rangle
$$


with

$$
\begin{aligned}
\left|n_{\alpha}\right\rangle & =\left(\phi_{\alpha}(x)\right)^{n_{\alpha}} \\
\left|n_{\beta}\right\rangle & =\left(\zeta_{\beta}(x)\right)^{n_{\beta}} \\
\otimes_{\alpha=1}^{\infty}\left|n_{\alpha}\right\rangle & =\left|n_{1}\right\rangle \otimes\left|n_{2}\right\rangle \otimes\left|n_{3}\right\rangle \otimes\left|n_{4}\right\rangle \otimes \ldots \\
\left\{n_{\alpha}\right\} & =n_{1} n_{2} n_{3} n_{4} n_{5} \ldots
\end{aligned}
$$

The time dependence of the modes $\phi_{\alpha}$ and $\zeta_{\beta}$ is just an arbitrary phase factor which can be set equal to unity. The time dependence arising from the relative phases is then contained in $A_{\left\{n_{\alpha}\right\}\left\{n_{\beta}\right\}}$. A scalar product $\left\langle\left\{n_{\alpha}\right\}\left\{n_{\beta}\right\} \mid\left\{n_{\gamma}\right\}\left\{n_{\delta}\right\}\right\rangle$ is defined by

$$
\left\langle\left\{n_{\alpha}\right\}\left\{n_{\beta}\right\} \mid\left\{n_{\gamma}\right\}\left\{n_{\delta}\right\}\right\rangle=\prod_{\alpha, \beta, \gamma, \delta=1}^{\infty} \delta_{n_{\alpha} n_{\gamma}} \delta_{n_{\beta} n_{\delta}}
$$

We refer to $\left|\left\{n_{\alpha}\right\}\left\{n_{\beta}\right\}\right\rangle$ as a (field) configuration. Introducing creation and annihilation operators $a_{\alpha}^{+}, a_{\alpha}, b_{\beta}^{+}, b_{\beta}$ in the usual way, one writes for the field configuration

$$
\left|\left\{n_{\alpha}\right\}\left\{n_{\beta}\right\}\right\rangle=\prod_{\alpha=1}^{\infty} \prod_{\beta=1}^{\infty}\left(a_{\alpha}^{+}\right)^{n_{\alpha}}\left(b_{\beta}^{+}\right)^{n_{\beta}}\left|\left\{n_{\alpha}=0\right\}\left\{n_{\beta}=0\right\}\right\rangle .
$$

The solution of the time dependent Schrödinger equation is

$$
|\Psi(t)\rangle=e^{-\mathrm{iH} t}|\Psi(t=0)\rangle
$$

where $\mathrm{H}$ is the Hamiltonian derived in Sects.3-5. The time dependent amplitude becomes

$$
A_{\left\{n_{\alpha}\right\}\left\{n_{\beta}\right\}}(t)=\left\langle\left\{n_{\alpha}\right\}\left\{n_{\beta}\right\}\left|e^{-\mathrm{iH} t}\right| \Psi(t=0)\right\rangle .
$$

The initial functional $|\Psi(t=0)\rangle$ can be expanded in the field configurations:

$$
|\Psi(t=0)\rangle=\sum_{\left\{n_{\gamma}\right\}\left\{n_{\delta}\right\}} B_{\left\{n_{\gamma}\right\}\left\{n_{\delta}\right\}}\left|\left\{n_{\gamma}\right\}\left\{n_{\delta}\right\}\right\rangle .
$$

For the amplitude one obtains then

$$
A_{\left\{n_{\alpha}\right\}\left\{n_{\beta}\right\}}(t)=\sum_{\left\{n_{\gamma}\right\}\left\{n_{\delta}\right\}} B_{\left\{n_{\gamma}\right\}\left\{n_{\delta}\right\}}\left\langle\left\{n_{\alpha}\right\}\left\{n_{\beta}\right\}\left|e^{-\mathrm{iH} t}\right|\left\{n_{\gamma}\right\}\left\{n_{\delta}\right\}\right\rangle .
$$

The CI matrix elements in the last equation have to be evaluated using the Hamiltonian $\mathrm{H}$ which consists of terms like $h_{\alpha \beta} a_{\alpha}^{+} a_{\beta}, v_{\gamma \delta} b_{\gamma}^{+} b_{\delta}, h_{\alpha \beta \gamma \delta} a_{\alpha}^{+} a_{\beta} b_{\gamma}^{+} b_{\delta}$ and their hermitian conjugate. The operation $a_{\kappa}^{+} a_{\lambda} b_{\mu}^{+} b_{\nu}\left|\left\{n_{\alpha}\right\}\left\{n_{\beta}\right\}\right\rangle$ is defined by Eq. (21). 


\subsubsection{Telegraph Signals ("Quantum Jumps”)}

Whereas the theory is relativistic, i.e., Lorentz invariant, the interesting solutions emerge in the non-relativistic limit. If the time dependent wave functional is calculated along the lines just outlined, one finds that configurations $\left|\left\{n_{\alpha}=1\right\}\left\{n_{\beta}=0\right\}\right\rangle$ restricted to four dimensional spacetime ("three dimensional configurations") become heavily entangled with configurations living essentially in eleven dimensional spacetime. The reason is that due to the high density of "gravonon configurations" (i.e. configurations where gravonons in the hidden dimensions have been excited, cf. Sect. 3.2) a particular four dimensional configuration will always be practically degenerate with gravonon configurations and, as it is well known, the Schrödinger equation will mix degenerate configurations even for vanishingly small interaction strength.

In the problem of diffusion of an adsorbed particle on a solid surface the Hamiltonian has been chosen as $[69,70]$ :

$$
\begin{aligned}
\mathrm{H}= & \mathrm{H}_{\text {gas atom }}+\mathrm{H}_{\text {graviton }}+\mathrm{H}_{\text {gas atom-graviton }} \\
= & E_{g_{1}} n_{g_{1}}+E_{g_{2}} n_{g_{2}}+E_{w_{1}} n_{w_{1}}+E_{w_{2}} n_{w_{2}}+\sum_{i=1}^{2} V_{l o c}^{g_{i}}\left(a_{g_{i}}^{+} a_{w_{i}}+a_{w_{i}}^{+} a_{g_{i}}\right) \\
& +\sum_{i=1}^{2}\left\{\varepsilon_{\text {grav }_{i}} b_{\text {grav }_{i}}^{+} b_{\text {grav }_{i}}+\sum_{k} \varepsilon_{k_{i}} b_{k_{i}}^{+} b_{k_{i}}\right. \\
& \left.+\sum_{k}\left[V_{\text {grav }_{i}, w_{i}} n_{w_{i}} b_{\text {grav }_{i}}^{+} b_{k_{i}}+V_{w_{i}, \text { grav }_{i}} n_{w_{i}} b_{k_{i}}^{+} b_{\text {grav }_{i}}\right]\right\} .
\end{aligned}
$$

The meaning of the symbols is: $n_{g_{i}}, n_{w_{i}}$ : gas particle field strength in the vibrationally ground and excited (parallel to the surface) core movement states and in the "warp resonance" where interaction with the gravonons occurs; $a_{i}^{+}, a_{j}$ : creation and annihilation operators for the gas particle field in the respective core movement states; $V_{l o c}^{g_{i}}$ : interaction strength between a gas particle state and the warp resonance; $\varepsilon_{g r a v}, \varepsilon_{k_{i}}$ : energy quanta of local and continuum gravonon fields; $b_{\text {gravi }}^{+}, b_{\text {grav }}$ : creation and annihilation operators for the local gravonon field; $b_{k_{i}}^{+}, b_{k_{i}}$ : creation and annihilation operators for the continuum gravonon field; $V_{\text {gravi }}, w_{i}$ : interaction between the gas particle and the gravonons within the warp resonance.

The field configurations taken into account are the following:

$$
\left\{n_{\alpha}\right\}=n_{g 1} n_{g 2} n_{w 1} n_{w 2}
$$

with $n_{g i}=0,1, n_{w i}=0,1$ for $i=1,2$.

$$
\left\{n_{\beta}\right\}=n_{k 1} n_{k 2}
$$

with $n_{k i}=0,1, \ldots, \infty$ for $i=1,2$.

The solution of the Schrödinger equation for this Hamiltonian results in the adparticle being fixed near a particular substrate atom as long as the excited gravonons 


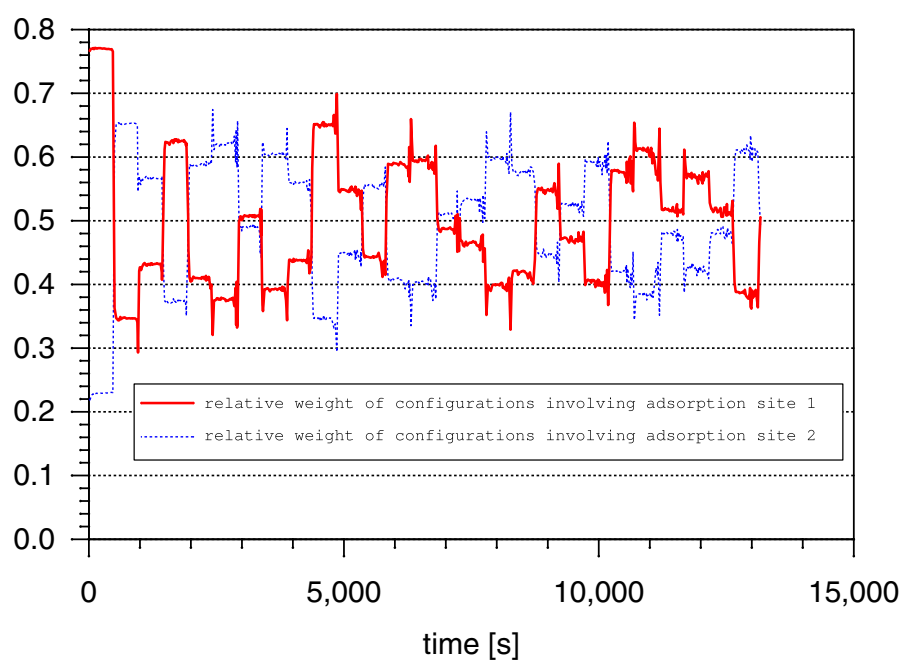

Fig. 1 Adsorbate telegraph-signal-like quantum jumps between two adsorption sites as a function of time obtained as a solution of the Schrödinger equation. The entanglement with the gravonon field is included. The quantity plotted is the sum of the squared coefficients of the field configurations containing gravonons at site 1 and site 2, respectively. The full curve (red) is site 1, the dashed curve (blue) is site 2 (Color figure online)

are moving around in the large hidden dimensions. If after the recurrence time the gravonons return to the four dimensional brane where they have been excited, the adparticle is suddenly free to leave this site. When arriving at a neighbouring site the adparticle will excite gravonons here and will be captured to this new site as long as the excited gravonons are moving around in the large hidden dimensions. This leads to the telegraph signal like adsorbate movement, which is observed in experiment. A calculation of this movement within the present theory is depicted in Fig. 1 and has been published in Ref. [70]. The analytical derivation of why and how telegraph signal like changes in the state of a quantum system, entangled with gravonons in the environment, occur, is presented in Ref. [71].

Flat two dimensional surfaces present ideal systems for experimental studies of gravonon coupling. They can be experimentally prepared with high accuracy. Their two dimensional translational symmetry is observed, e.g. for surface states on the (111) faces of $\mathrm{Cu}, \mathrm{Ag}$ and $\mathrm{Au}$ and for well ordered adsorbate layers. Delocalized movement is observed, for instance, for electrons in the surface state on $\mathrm{Cu}(111)$ and it has also been predicted as solution of Schrödinger's equation in four dimensional spacetime for weakly adsorbed atoms and molecules (e.g. rare gas atoms). However, delocalized adsorbate movement has not been verified experimentally, as it was demonstrated for instance in the STM experiment of Eigler with adsorbed Xe on Ni(110) [34]. The reason is that the substrate surface provides a close packed array of massive centers (the substrate atoms). A massive adsorbate has always a short distance from the nearest massive substrate center and, therefore, substantial coupling to the gravonons which leads to entanglement. Thus the entanglement is between the two dimensional surface, existing in four dimensional spacetime, and the gravonons in eleven dimensional spacetime. 
In fact single atom diffusion on cold, flat surfaces with alternating localization and telegraph signal like motion (sudden jumps) cannot be explained either by decoherence theory or spontaneous collapse theories. Of course, the interpretation within CQM can be reconciled with these experimental observations, if localization and jumps are interpreted as collapses of unknown origin and reason.

\subsubsection{The Chooser Mechanism ("Collapse”)}

The scattering of a matter field from a double slit and the "wave-particle duality" observed in these experiments is in our theory interpreted as follows. If there is a site on the screen which is energetically degenerate with the initial wave functional $|Q 0\rangle=|\Psi(t=0)\rangle$ the configuration $\left|K \kappa_{\text {proj }}\right\rangle$ with the scalar matter field $\phi$ having significant strength on this site will get strongly entangled with the degenerate gravonon configurations. As the excitations of the gravonons in the hidden dimensions depend on the matter field being extremely localized near the chosen site, the matter field strength cannot drift away as long as the excited gravonons are moving around in the large hidden dimensions. If this takes time long enough for other physical-chemical processes to be initiated, it will result in an experimentally detectable event. Such an event would be called "collapse" in the framework of CQM.

To treat this situation theoretically in a simplified manner we set up the Hamiltonian $\mathrm{H}$ in matrix form

\begin{tabular}{llll}
\hline & $|Q 0\rangle$ & $|R 0\rangle$ & $\left|K \kappa_{\text {proj }}\right\rangle$ \\
\hline$\langle Q 0|$ & 0 & $V$ & 0 \\
$\langle R 0|$ & $V$ & 0 & $\mathrm{~W}$ \\
$\left\langle K \kappa_{\text {proj }}\right|$ & 0 & $\mathrm{~W}$ & 0 \\
\hline
\end{tabular}

Figure 2 illustrates the many-particle states involved and their interactions. $|R 0\rangle=|R\rangle \otimes\left|0_{\text {grav }}\right\rangle$ is a a state where the matter field is localized on the screen but not yet entangled with the gravonons. In the state $\left|K \kappa_{\text {proj }}\right\rangle$ the matter field experiences the interaction with the gravitational field. All three states are degenerate at energy $E=0$ which defines the energy zero. The interaction $V$ allows the matter field to penetrate into the localized state $|R 0\rangle$ on the screen and $W$ is the potential connecting $|R 0\rangle$ to the resonance $\left|K \kappa_{\text {proj }}\right\rangle$ where gravitational interaction becomes important.

Diagonalizing the Hamiltonian leads to the characteristic equation

$$
\operatorname{det}(E-\mathrm{H})=E^{3}-\left(W^{2}+V^{2}\right) E=0
$$

which has the solutions $E_{o}=0, E_{ \pm}= \pm \sqrt{W^{2}+V^{2}}$. Only the eigenstate $|00\rangle$ of energy zero is of interest in the following. It has the coefficients

$$
\begin{aligned}
& C_{Q 0}=\frac{W}{\sqrt{V^{2}+W^{2}}} \\
& C_{R 0}=0
\end{aligned}
$$




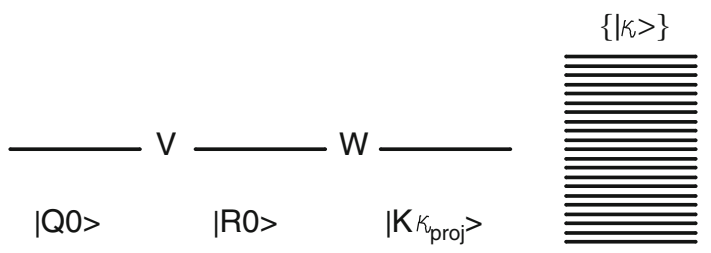

Fig. 2 Field configurations and their interactions: $|Q 0\rangle=|Q\rangle \otimes\left|0_{\text {grav }}\right\rangle$ initial field configuration with the gravitational field in the ground state and the matter field in the source; $|R 0\rangle=|R\rangle \otimes\left|0_{\text {grav }}\right\rangle$ with the matter field localized on the screen but not yet entangled with the gravonons, which are in the ground state; $\left|K \kappa_{\text {proj }}\right\rangle$ with the matter field localized on the screen and a gravonon state projected out of the gravonon band $\{|\kappa\rangle\}$ according to the criterion of strongest interaction

$$
C_{K \kappa_{\text {proj }}}=\frac{V}{\sqrt{V^{2}+W^{2}}}
$$

In the limit where the coupling to the gravonons becomes very weak $\left(\frac{W}{V} \rightarrow 0\right) C_{Q 0}=$ 0 and $C_{K \kappa_{p r o j}}=1$. The coefficients of the "zero-state" $|00\rangle$ in the eigenstates of the total system become then approximately

$$
\begin{aligned}
\langle 00 \mid K \kappa+\rangle & =\left\langle 00\left|\mathrm{G}^{+}\left(\epsilon_{\kappa}\right)\left(W_{\text {grav }}+V+W\right)\right| K \kappa\right\rangle \\
& \approx\left\langle K \kappa_{\text {proj }}\left|\mathrm{G}^{+}\left(\epsilon_{\kappa}\right)\left(W_{\text {grav }}+V+W\right)\right| K \kappa\right\rangle \\
& =\left\langle K \kappa_{\text {proj }}\left|\mathrm{G}^{+}\left(\epsilon_{\kappa}\right)\right| K \kappa_{\text {proj }}\right\rangle\left\langle K \kappa_{\text {proj }}\left|W_{\text {grav }}\right| K \kappa\right\rangle \\
& =G_{K \kappa_{\text {proj }}}^{+}\left(\epsilon_{\kappa}\right) W_{\kappa}
\end{aligned}
$$

where $\mathrm{G}^{+}$is the Green operator, $W_{\text {grav }}$ is the gravitational potential, $G_{K \kappa_{p r o j}}^{+}=$ $\left\langle K \kappa_{\text {proj }}\left|\mathrm{G}^{+}\right| K \kappa_{\text {proj }}\right\rangle$ and $W_{\kappa}=\left\langle K \kappa_{\text {proj }}\left|W_{\text {grav }}\right| K \kappa\right\rangle .|\kappa\rangle$ is one of the states in the gravonon band, generating $|\kappa+\rangle$ after the interaction with the matter field is included. $\epsilon_{\kappa}$ is the eigenenergy of the eigenstate $|K k+\rangle$. We have here applied the Lippmann-Schwinger equation [72] to construct the eigenstates $|K \kappa+\rangle$. The Green function $G_{K \kappa_{p r o j}}^{+}$has the form

$$
G_{K \kappa_{p r o j}}^{+}\left(\epsilon_{\kappa}\right)=\frac{1}{\epsilon_{\kappa}-\alpha\left(\epsilon_{\kappa}\right)+\mathrm{i} \Gamma\left(\epsilon_{\kappa}\right)}
$$

where $\alpha$ and $\Gamma$ are the real and imaginary parts of the self-energy, respectively, with

$$
\begin{aligned}
\Gamma\left(\epsilon_{k}\right) & =\pi \sum_{\kappa}\left|W_{\kappa}\right|^{2} \delta\left(E-\epsilon_{\kappa}\right) \\
& =\pi \frac{U^{2}}{\Delta} \text { if }\left|\epsilon_{k}\right|<\Delta .
\end{aligned}
$$

The last line indicates an approximation where $W_{\kappa}=\frac{U}{\sqrt{N}}$ is non-zero and energy independent over an energy range of length $\Delta$ and zero otherwise. $N$ is the number 
of gravonons in the energy interval $\Delta$. Defining the density $\rho_{\epsilon}$ of gravonon levels at $\epsilon_{\kappa}=0$

$$
\begin{aligned}
\frac{\rho_{\epsilon}}{N} & =\frac{1}{\Delta} \\
& =-\frac{1}{\pi} \operatorname{ImG}_{K \kappa_{p r o j}}^{+} \\
& =\frac{1}{\pi \Gamma}
\end{aligned}
$$

yields $\Delta=\pi \Gamma$. In order to study the time dependence due to the coupling to the gravonons we start from the formal solution of the time dependent Schrödinger equation

$$
\begin{aligned}
|\Psi(t)\rangle & =e^{-\mathrm{iH} t}|00\rangle \\
& =\sum_{\kappa} \mathrm{e}^{-\mathrm{i} \epsilon_{\kappa} t}|K \kappa+\rangle\langle K \kappa+\mid 00\rangle \\
& =\sum_{\kappa} \mathrm{e}^{-\mathrm{i} \epsilon_{\kappa} t}|K \kappa+\rangle G_{K \kappa_{p r o j}}^{-}\left(\epsilon_{\kappa}\right) W_{\kappa} \\
& =\sum_{\kappa} \mathrm{e}^{-\mathrm{i} \epsilon_{\kappa} t}|K \kappa+\rangle \frac{W_{\kappa}}{\epsilon_{\kappa}-\alpha\left(\epsilon_{\kappa}\right)-\mathrm{i} \Gamma\left(\epsilon_{\kappa}\right)}
\end{aligned}
$$

where we assumed $W_{\kappa}$ to be real. For the projection $\left\langle K \kappa_{\text {proj }} \mid \Psi(t)\right\rangle$ we obtain:

$$
\left\langle K \kappa_{p r o j} \mid \Psi(t)\right\rangle=\sum_{\kappa} \mathrm{e}^{-\mathrm{i} \epsilon_{\kappa} t}\left\langle K \kappa_{p r o j} \mid K \kappa+\right\rangle \frac{W_{\kappa}}{\epsilon_{\kappa}-\alpha\left(\epsilon_{\kappa}\right)-\mathrm{i} \Gamma\left(\epsilon_{\kappa}\right)} .
$$

From Eq. (38), with $W_{\kappa}$ assumed to be independent of $\epsilon_{\kappa}$, we have:

$$
\left\langle K \kappa_{\text {proj }} \mid K \kappa+\right\rangle W_{\kappa}=\frac{U}{N} .
$$

Inserting in Eq. (44) we obtain

$$
\left\langle K \kappa_{p r o j} \mid \Psi(t)\right\rangle=\frac{U}{N} \sum_{\kappa} \frac{\mathrm{e}^{-\mathrm{i} \epsilon_{\kappa} t}}{\epsilon_{\kappa}-\alpha\left(\epsilon_{\kappa}\right)-\mathrm{i} \Gamma\left(\epsilon_{\kappa}\right)}
$$

where $\left\langle K \kappa_{\text {proj }} \mid K \kappa+\right\rangle=\frac{1}{\sqrt{N}}$ is assumed to be independent of $\epsilon_{\kappa}$. We have to emphasize at this point that the summation index $\kappa$ is not the graviton wave vector. The sum runs over the energy levels of the gravonon states (cf. Sect.3.2). The sum in Eq. (46) can therefore be transformed into an integral by writing

$$
\left\langle K \kappa_{\text {proj }} \mid \Psi(t)\right\rangle=\frac{U}{N} \int_{-\infty}^{\infty} \mathrm{d} \epsilon_{\kappa} \rho_{\epsilon} \frac{\mathrm{e}^{-\mathrm{i} \epsilon_{\kappa} t}}{\epsilon_{\kappa}-\alpha\left(\epsilon_{\kappa}\right)-\mathrm{i} \Gamma\left(\epsilon_{\kappa}\right)} .
$$


( $\rho_{\epsilon}$, the level density of gravonon states, is constant according to Eq. (40).) We assume $\alpha$ and $\Gamma$ to be independent of energy. The time dependent wave functional $|\Psi(t)\rangle$ and its component involving the localized matter state $|K\rangle$ is then obtained by integration yielding [73]

$$
\left\langle K \kappa_{\text {proj }} \mid \Psi(t)\right\rangle=\mathrm{i} \pi \frac{U}{N} \rho_{\epsilon} \mathrm{e}^{-\Gamma t}=\mathrm{i} \pi \frac{U}{\Delta} \mathrm{e}^{-\Gamma t},
$$

where in the weak interaction limit we assumed $\alpha \rightarrow 0$ and $\rho_{\epsilon}=\frac{N}{\Delta}$ (Eq. 40).

In order to clarify the complete character of the state $|\Psi(t)\rangle$ we have also to calculate its projections on $|R 0\rangle,|Q 0\rangle$ and $|K \lambda+\rangle$.

$$
\begin{aligned}
\langle R 0 \mid K \kappa+\rangle & =\left\langle R 0\left|G^{o}\left(W_{\text {grav }}+V+W\right)\right| K \kappa+\right\rangle \\
& =G_{R 0}^{o}\left\langle R 0\left|\left(W_{\text {grav }}+V+W\right)\right| K \kappa+\right\rangle \\
& =G_{R 0}^{o}\left\langle R 0\left|\left(W_{\text {grav }}+V+W\right)\right| K \kappa_{\text {proj }}\right\rangle\left\langle K \kappa_{\text {proj }} \mid K \kappa+\right\rangle \\
& =\frac{W}{\sqrt{N}} \lim _{\epsilon \rightarrow 0} \frac{1}{\epsilon_{\kappa}+\mathrm{i} \epsilon} \\
& =\frac{W}{\sqrt{N}}\left[\mathcal{P}\left(\frac{1}{\epsilon_{\kappa}}\right)-\mathrm{i} \pi \delta\left(\epsilon_{\kappa}\right)\right]
\end{aligned}
$$

Using this in Eq. (43) yields then with $W_{k}=U / \sqrt{N}$ and $\rho_{\epsilon}=N / \Delta$

$$
\begin{aligned}
&\langle R 0 \mid \Psi(t)\rangle=\pi \frac{U W}{\Delta(\Gamma-\mathrm{i} \alpha)} \\
&\langle Q 0 \mid K \kappa+\rangle=\left\langle Q 0|G| K \kappa_{\text {proj }}\right\rangle\left\langle K \kappa_{\text {proj }}\left|W_{\text {grav }}\right| K \kappa\right\rangle \\
&=\frac{U}{\sqrt{N}}\left\langle Q 0|G| K \kappa_{\text {proj }}\right\rangle
\end{aligned}
$$

Inserting the Born series

$$
G=G^{o}+\sum_{n=0}^{\infty}\left(G^{o}(V+W) G^{o}\right)^{n}
$$

one has

$$
\langle Q 0 \mid K \kappa+\rangle=\frac{W}{\sqrt{N}} \sum_{n=0}^{\infty}\left(\left\langle Q 0\left|G^{o}\right| Q 0\right\rangle\langle Q 0|V| R 0\rangle\left\langle R 0\left|G^{o}\right| K \kappa_{\text {proj }}\right\rangle\right)^{n}
$$

which is zero at every order and hence $\langle Q 0 \mid K \kappa+\rangle=0$. Requiring unitarity and setting $\alpha=0$ one gets 


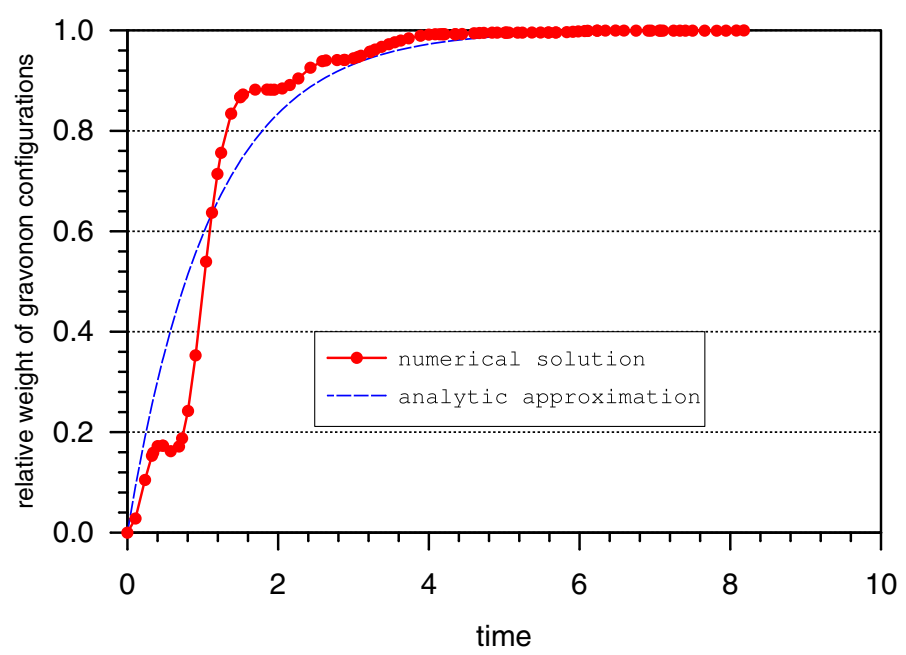

Fig. 3 The chooser at work: the numerical solution of the model of Sect.2.2.2 in the basis displayed in Fig. 2 (full curve) in comparison to the analytical approximation Eq. (61) (dashed curve)

$$
\begin{aligned}
\sum_{\lambda}|\langle K \lambda+\mid \Psi(t)\rangle|^{2}= & 1-\left|\left\langle K \kappa_{\text {proj }} \mid \Psi(t)\right\rangle\right|^{2} \\
& -|\langle R 0 \mid \Psi(t)\rangle|^{2}-|\langle Q 0 \mid \Psi(t)\rangle|^{2} \\
= & 1-\pi^{2}\left[\frac{U^{2}}{\Delta^{2}} e^{-2 \Gamma t}+\left(\frac{U W}{\Delta \Gamma}\right)^{2}+0\right] \\
& \approx 1-e^{-2 \Gamma t}-\frac{W^{2}}{U^{2}}
\end{aligned}
$$

The last line is valid, because from Eq. (38) $\frac{U^{2}}{\Delta}=\Gamma / \pi$ and $\Delta=\pi \Gamma$ (Eq. 40).

Neglecting off-shell components, the initial state $|Q 0\rangle$, by propagating through the nano lattice (or double slit), becomes the state $|00\rangle$, which for weak gravitational interaction strength merges into the state $\left|K \kappa_{\text {proj }}\right\rangle$ and then is completely extinguished with time according to Eq. (48). The whole field strength is captured in the states $\{|K \kappa+\rangle\}$, representing a localized matter field, strongly entangled with gravonon states, which live mainly in the hidden dimensions.

In Fig. 3 the sum of the weights of the gravonon configurations (squared coefficients) in the wave functional shows that as the system, displayed in Fig. 2, develops with time the weight of the field configurations with gravonon components increases, tending to 1 . This means that the matter field is localized and a "collapse" occurs on a single site $|K\rangle$ where the entanglement with the gravonons is effective. The oscillations in the exact numerical calculation arise, as the off-shell states with $E_{ \pm}$are also mixed in because of the finite energy spread of the gravonons.

A closer inspection including exact numerical result shows that energetical degeneracy between the chooser and the initial state is not a sufficient condition. The gravonon structure is a further condition for the chooser. At time $t$ the wave packet describ- 
ing the screen contains for every site, i.e. for every substrate atom, the momentary configuration, in particular the position distribution and the corresponding gravonon configuration. Only for one site it can be expected that it meets the condition of being the choice for adsorbate capture. This point is detailed in Ref. [74]. The wave packet describing the screen is perfectly determined and if the experimenters were able to prepare it accurately, they could determine the site where the diffracting molecule sticks. But for practical reasons this is impossible and, therefore, the choice of the site appears random. In principle, however, an experimenter would be able to determine in physical space which choice is made. The situation is similar to classical mechanics where an accurate knowledge of all initial conditions would allow a precise prediction of all trajectories. Uncertainties in the knowledge of the initial conditions, however, can make the trajectories appear random and even chaotic.

In the present theory the experimentally detectable event is uniquely determined already at the moment of the emission of the matter field quantum from the source, because energetical degeneracy is needed and, as outlined in Sect.2.1.1, due to the weakness of the interaction there is only one site of suitable energy and gravonon structure available on the screen.

\subsubsection{Beables and Non-locality}

Following Bell [75-77] we exclude the notion of observables in favour of beables. The local beables in Bohmian mechanics are the particle positions. As time elapses the particle positions follow a trajectory which in the double slit experiment passes through one or the other slit. In collapse models the probability amplitude for finding the particle at particular positions is spread throughout space. In these models the individual spot on the screen does not reflect where the particle position was just before the spot forms. An ontology for collapse models can then be constructed by either interpreting the modulus squared of the probability amplitude as a matter density or by the so called flash ontology where local beables, associated with particles, exist only when a collapse of the wave function occurs. The flash ontology permits a completely relativistic version of the Ghirardi-Rimini-Weber theory [78]. The beables [75-77] of our theory are the field configurations where matter fields extremely localized in three dimensional space are entangled to gravonons moving in 11 dimensional spacetime. These beables emerge in the non-relativistic limit within a preferred Lorentz frame, corresponding to the rest frame of the investigated system, the laboratory and the experimenter.

Unlike CQM where few "observables" have to be chosen from an infinite set of hermitian operators by using criteria which lie outside the realm of CQM, namely by being found suitable for the interpretation of experiments, the beables which define measurement are uniquely defined within our theory, based on the Schrödinger equation.

It is interesting that our definition of a beable is compatible with Kant's ideas about observations [79]. According to Kant, knowledge rests on our sensual contact with the world, and our sensibility has its limits. The sensual limits Kant called the forms of intuition. He argued that the forms are space and time [80]. All intelligible experiences involve the general concept of the objective world. The general concept is a sponta- 
neous product of human understanding. The general concept of the objective world is associated with the category of substance which in our theory is represented by the beables, consisting of matter fields localized in four dimensional spacetime and entangled to gravonons in eleven dimensional spacetime. Kant makes great effort to justify the objective validity of the general concept. The most important argument is the "transcendental deduction". We have experiences, this requires certain conditions which, therefore, are real (a priori). Einstein commented on this point as follows: 'This conceptual model refers precisely to the "real" (by definitions), and every further question concerning the "nature of the real" appears empty' [81]. Kant's general concepts are intrinsic to all objective experiences as their presuppositions, hence they are a priori. In the general conceptual structure of experiences, the forms of intuition ["Anschauung"] are incorporated as the schematization that introduces particulars. According to our understanding this Kantian construction resembles the way our beables, i.e. our category of substance, are related to the eleven dimensional spacetime, i.e. the forms of intuition in our theory. Although it is not possible for us to gain a complete intuition of spaces with more than three dimensions we are able to investigate all details we would experience in spaces of higher dimensions. The intuitive nature of our beables is in marked contrast to the artificial constructs which are sometimes necessary in CQM to define e.g. the measured expectation values of the electromagnetic field (see Ref. [80, Note 43]).

The transition from configuration space to the three dimensional space experienced by the experimenter is unambiguously provided by the localization in three dimensional space of the matter field. The beables are experienced in our three dimensional brane space as matter fields localized at different space points. Beables localized in this sense at different points in three dimensional brane space can appear simultaneously, if we describe the three dimensional space in the rest frame.

Consider Einstein-Podolsky-Rosen paradox. The particle with spin up (just "up" in the following) is simultaneously at Alice's and Bob's place, respectively, as is the particle with spin down (just "down" in the following). If "up" is chosen (by whatever chooser Alice has) at Alice's place, i.e. the "up" field becomes localized and entangled to gravonons there, only "down" remains in a delocalized wave packet which is simultaneously at Alice's and Bob's place, respectively. This becomes true at the very moment when "up" is chosen at Alice's place, also if Alice and Bob are separated by thousands of kilometers. This is referred to as non-locality.

Hence, non-locality is a phenomenon which persists in our theory, as it does in the Copenhagen interpretation.

The fact that the beables only emerge in the non-relativistic limit and are neither defined nor empirically accessible for relative velocities (between experimenter and physical event), approaching the velocity of light, resolves the apparent paradox of "real collapses in Minkowski spacetime" [82]. According to this paradox beables which appear simultaneously in one Lorentz frame appear at different times in another Lorentz frame. This difference in time is noticeable only, if the relative velocity of the two Lorentz frames approaches the velocity of light.

As both the experimenter and the physical event which she is to observe consist of beables, according to the present theory the beables representing "collapses" and/or "quantum jumps" are not empirically accessible as such by the experimenter, if the 
physical event moves with a velocity near to the velocity of light relative to the experimenter. This is associated with the fact that our theory does not contain "collapses" or "quantum jumps" as additional processes compared to CQM, where these processes occur in all Lorentz frames immediately. In our theory collapses are replaced by deterministic localization processes which can occur and switch in a telegraph signal like way. These telegraph signal like processes occur in the rest frame of the experiment within a very short time, but they are not immediate. If the observer moves with a high velocity relative to the experimental setup, the telegraph signal like processes slow down and can no longer represent "collapses" or "quantum jumps" of the CQM type.

This is the well established time dilatation which is present in our Lorentz invariant theory. As is common in popular presentations of special relativity this can be described in the way that for high relative velocity the observer experiences an increased mass of the matter and gravonon fields which leads to a slower movement. Processes, which in the rest frame of the experimental setup appear immediate and simultaneous, are realized in a boosted Lorentz frame as blurred in time and simultaneity of the experiments is no longer defined.

We can elaborate on this aspect in the following way. Assume that Alice and Bob perform an EPR-experiment [83] in earth bound laboratories and measure at distant locations but simultaneously the spins of two particles bound in a singlet state, thus verifying the established non-locality emanating from the Schrödinger equation. At the moment when they measure the spins (i.e. simultaneously) each one sends a light signal to a rocket, which moves with a velocity near to the velocity of light above them in the direction from Bob to Alice. The observer in the rocket registers the light signal from Alice earlier than that from Bob and therefore concludes in the rocket bound rest frame that the two measurements have not been performed simultaneously.

Why does the apparent paradox in Minkowski space exist? This is the kind of reasoning that leads to it. The observer in the rocket would say that in the time interval, which starts, when she "sees" that Alice measures the spin of particle 1, and ends, when she "sees" that Bob measures the spin of particle 2, the spin of particle 2 is not determined. In this time interval it is therefore not guaranteed that the system, consisting of the two particles, is in a singlet state, i.e., that the total spin is conserved. This means, the observer in the rocket finds that the rules of CQM are violated (cf. e.g. [84]). The Schrödinger equation, however, requires that there can be no temporal gap between the measurement of the spin of particle 1 and the time, when the spin of particle 2 acquires a definite value. One further reasons that the rest frame of the earth and of the rocket are connected by a Lorentz transformation and, hence, are fully equivalent. It is deduced then that the two events (measuring the spin of particle 1 and measuring the spin of particle 2) are real and immediate and their existence is independent of the chosen Lorantz frame. However, the existence of an event in the rocket, (i.e. the recording of the photons in the rocket bound reference frame), and events in the earth bound reference frame, (i.e. the measurement of the spin of particle 1 by Alice simultaneously with the measurement of the spin of particle 2 by Bob), are associated with different beables, hence with further events. The fact that events additional and different from Alice's and Bob's measurements have to occur (the light signals hitting the retina or some registration device and initiating a physical-chemical process) is not considered to be of importance and is not discussed. 
In our theory, however, every measurement is a beable of the kind defined at the end of the first paragraph in the present chapter. If the rocket stays far off the laboratories on earth, the judgement of the observer in the rocket has to rely on the information contained in the light signal as born out by the beables it generates in the rocket. The light emission process which is immediate in the rest frame of the experimental setup appears extended in time in the rocket.

The reasoning presented here is only valid, because in the first place the beables do not emerge independent of the reference frame and in the second place measurement is a consequence of the Schrödinger equation. In our theory all events, i.e. beables, in the rest frame of the rocket (including those constituting the observer) emerge from the Schrödinger equation in the rest frame of the observer. Therefore, there is no paradox, i.e., no contradiction either to the observed facts, or to the symmetry of Lorentz invariance, or to the predictions of the Schrödinger equation.

In our theory the wave functional $|\Psi\rangle$ yields a complete description of the world. The beables, which constitute both observables and measurements, are generated by solving the Schrödinger equation for the wave functional in the non-relativistic limit. All measurements of various physical quantities can be traced back to determining the location of the particles [85].

\subsubsection{A Remark on Entropy}

Our world wave functional is unique and a solution of the Schrödinger equation. Therefore the von Neumann entropy associated with this wave functional is zero. Entropy, however, in its statistical and /or thermodynamic applications is clearly a quantity which should be defined in four dimensional spacetime. As the events in four dimensional spacetime are entangled to events in the seven hidden dimensions, an evaluation of entropy in four dimensional spacetime would be conventionally tried by using the entanglement entropy.

To define entropy in our theory by means of the density matrix is, however, not possible, because $|\Psi\rangle$ does not have the meaning of a probability amplitude. It is rather a description of the various fields (matter, electromagnetic, gravitational) in configuration space. A configuration $\left|\left\{n_{\alpha}\right\}\left\{n_{\beta}\right\}\right\rangle$ is a distribution of the fields in 10 dimensional space.

Entropy has then to be defined as being proportional to the logarithm of the number of microscopic field configurations, representing a macroscopic field configuration. In our case this is then the logarithm of the number of initial conditions (source of matter field, number of gravonon structures $\left\{n_{\beta}\right\}$ ) which lead to the state $\left|n_{\alpha}\right\rangle$ for the matter field in four dimensional spacetime.

Compared to CQM we switch from a probabilistic interpretation of $\left|\left\{n_{\alpha}\right\}\right\rangle$ to a statistical interpretation. Beables can be experienced in the four dimensional world in exactly the same way, if they differ only by slight variations in the gravonon configuration in the hidden seven dimensions. There are numerous such variations for each beable, which appear identical in their four dimensional part. It is principally impossible for us to know, if a particular beable will be realized. Statistics means that we can predict the occurrence of a particular beable, if we start from a precisely defined 
initial condition. Due to limited experimental resolution we have a sample of many initial conditions which permits only statistical statements.

Clearly the entropy increases steadily as further beables are created. The entanglement between the matter fields and the gravonons persists forever, because there is nothing like a "collapse" of the total wave functional or any other external influence. The total (world) wave functional develops continuously and deterministically according to the dynamics dictated by the Schrödinger equation. The second law is thus a consequence of our theory and need not be introduced as an additional postulate.

\section{Effective Hamiltonian for Matter-Graviton Interaction}

In this section the effective Hamiltonian of our EQM is derived from first principles to describe the matter-graviton interaction. The Hamiltonian describes the matter field as a scalar boson field (denoted $\phi$ later on) in interaction with a gravonon field (denoted $\zeta_{00}$ later on) in the presence of gravitational interaction. This derivation shows how the gravonon field emerges from linearized general relativity.

\subsection{Weak Field Gravity}

The following introduction to gravity as a field theory is in the spirit of Zee [63]. Units are $\hbar=\mathrm{c}=1$.

The Einstein-Hilbert action for gravity in $N$ spacetime dimensions is

$$
S_{\text {grav }}=\frac{1}{16 \pi G^{(N)}} \int \mathrm{d}^{N} x \sqrt{-g} R,
$$

where $g=\operatorname{det} g_{\mu \nu}$ denotes the determinant of the curved metric $g_{\mu \nu}$ of spacetime, $R$ is the scalar curvature, and $G^{(N)}$ is the gravitational constant in $N$ dimensional spacetime. The sign convention varies widely in the literature [86], we use the sign convention of [63].

In addition to gravity there are matter fields embedded in curved spacetime, for instance the electromagnetic field which lives only in four dimensional spacetime, as contributions $S_{\text {matter }}$ to the total action:

$$
S_{\text {tot }}=S_{\text {grav }}+S_{\text {matter }}
$$

$S_{\text {tot }}$ is assumed to describe the real world, if the theory is properly quantized. Quantization is only possible for weak gravitation. This is the procedure adopted here.

Alternatively one could treat quantum fields in a classical gravitational background. This is, however, a semi-classical approximation, which does not define a unique vacuum state and/or a unique world wave function. It would not allow to describe the entanglement of matter fields with gravitons which is proposed to contribute in a significant way to effects observed in experiment. Therefore the semi-classical theory is not followed here. 
The stress-energy tensor of the matter fields is derived from the action $S_{\text {matter }}$ :

$$
\begin{aligned}
T^{\mu \nu}(x) & =-\frac{2}{\sqrt{-g}} \frac{\delta S_{\text {matter }}}{\delta g_{\mu \nu}(x)} \\
T_{\mu \nu}(x) & =-\frac{2}{\sqrt{-g}} \frac{\delta S_{\text {matter }}}{\delta g^{\mu \nu}(x)} .
\end{aligned}
$$

$x$ is a $N$-dimensional vector denoting a point in $N$ dimensional spacetime. The signature is $(+,-,-,-,-,-,-,-, \ldots,-)$. These equations are valid in general. The weak field limit is defined by a linear deviation from the Minkowski metric $\eta_{\mu \nu}$ :

$$
g_{\mu \nu}=\eta_{\mu \nu}+h_{\mu \nu}
$$

Our philosophy is that this form of the metric is the correct one for the physics we want to investigate. Expanding $S_{\text {matter }}$ to first order in $\delta g_{\mu \nu}=h_{\mu \nu}$

$$
S_{\text {matter }}\left(h_{\mu \nu}\right)=S_{\text {matter }}\left(h_{\mu \nu}=0\right)+\delta S_{\text {matter }}
$$

and plugging in $\delta S_{\text {matter }}$ from Eq. (64) one obtains:

$$
\begin{aligned}
\delta S_{\text {matter }} & =-\int \mathrm{d}^{N} x \sqrt{-g} \frac{1}{2} h_{\mu \nu} T^{\mu \nu} \\
& =-\int \mathrm{d}^{N} x \sqrt{-g} \frac{1}{2} h^{\mu \nu} T_{\mu \nu} .
\end{aligned}
$$

$\sqrt{-g}$ is expanded as

$$
\sqrt{-g} \approx 1+\frac{\partial \sqrt{-g}}{\partial h^{\mu \nu}} h^{\mu \nu} .
$$

Using the following general relationship (Palatini method)

$$
\frac{\partial \sqrt{-g}}{\partial g^{\alpha \beta}}=-\frac{1}{2} \sqrt{-g} g_{\alpha \beta}
$$

together with

$$
\frac{\partial \sqrt{-g}}{\partial g^{\alpha \beta}}=\frac{\partial \sqrt{-g}}{\partial h^{\alpha \beta}}
$$

and $\sqrt{-g}=1$, we obtain:

$$
\begin{aligned}
\frac{\partial \sqrt{-g}}{\partial h^{\alpha \beta}} & =-\frac{1}{2}\left(1+\frac{\partial \sqrt{-g}}{\partial h^{\mu \nu}} h^{\mu \nu}\right)\left(\eta_{\alpha \beta}+h_{\alpha \beta}\right) \\
& =-\frac{1}{2} \eta_{\alpha \beta}-\frac{1}{2} h_{\alpha \beta}-\frac{1}{2} \frac{\partial \sqrt{-g}}{\partial h^{\mu \nu}} h^{\mu \nu} \eta_{\alpha \beta}-\frac{1}{2} \frac{\partial \sqrt{-g}}{\partial h^{\mu \nu}} h^{\mu \nu} h_{\alpha \beta}
\end{aligned}
$$




$$
\approx-\frac{1}{2} \eta_{\alpha \beta}-\frac{1}{2} h_{\alpha \beta}+\frac{1}{4}\left(\eta_{\mu v}+h_{\mu v}\right) h^{\mu \nu} \eta_{\alpha \beta}+\frac{1}{4}\left(\eta_{\mu \nu}+h_{\mu v}\right) h^{\mu v} h_{\alpha \beta}
$$

Omitting terms of higher order than linear in $h_{\alpha \beta}$ and plugging this into Eq. (68) yields

$$
\sqrt{-g}=1-\frac{1}{2} \eta_{\mu \nu} h^{\mu \nu}-\frac{1}{2} h_{\mu \nu} h^{\mu \nu}+\frac{1}{4} \eta_{\alpha \beta} h^{\alpha \beta} \eta_{\mu \nu} h^{\mu \nu}
$$

Inserting this in Eq.(67) we obtain, neglecting the last two terms in the previous equation:

$$
\delta S_{\text {matter }}=-\int \mathrm{d}^{N} x \frac{1}{2} h_{\mu \nu} T^{\mu \nu}\left(1-\frac{1}{2} \eta_{\alpha \beta} h^{\alpha \beta}\right) .
$$

A matter field is any field that is not the graviton field. Imposing the harmonic gauge condition (cf. refs. [63,87])

$$
\partial_{\mu} h_{v}^{\mu}=\frac{1}{2} \partial_{\nu} h_{\lambda}^{\lambda}
$$

$\left(h_{v}^{\mu}=h_{\nu \alpha} g^{\alpha \mu}\right)$ the action for the weak field gravity becomes (Ref. [63]):

$$
\begin{aligned}
S_{w f g}= & S_{g r a v}\left(g_{\mu \nu}=\eta_{\mu \nu}+h_{\mu \nu}\right)+\delta S_{\text {matter }} \\
= & \int \mathrm{d}^{N} x \frac{1}{2}\left[\frac{1}{32 \pi G^{(N)}}\left(\partial_{\lambda} h^{\mu \nu} \partial^{\lambda} h_{\mu \nu}-\frac{1}{2} \partial_{\lambda} h_{\mu}^{\mu} \partial^{\lambda} h_{\mu}^{\mu}\right)\right. \\
& \left.-h_{\mu \nu} T^{\mu \nu}\left(1-\frac{1}{2} \eta_{\alpha \beta} h^{\alpha \beta}\right)\right] .
\end{aligned}
$$

The total action is then:

$$
S_{\text {tot }}=S_{w f g}+S_{\text {matter }}\left(h_{\mu \nu}=0\right) \text {. }
$$

Varying Eq. (77) with respect to $h_{\mu \nu}$ we obtain the textbook result for the EulerLagrange equation of motion (Ref. [63]):

$$
\partial^{2} h_{\mu \nu}=-16 \pi G^{(N)}\left(\tilde{T}_{\mu \nu}-\frac{1}{2} \eta_{\mu \nu} \tilde{T}\right)
$$

where we define:

$$
\begin{aligned}
\tilde{T}_{\mu \nu} & =T_{\mu \nu}\left(1-\frac{1}{2} \eta_{\alpha \beta} h^{\alpha \beta}\right) \\
\tilde{T} & =T\left(1-\frac{1}{2} \eta_{\alpha \beta} h^{\alpha \beta}\right)
\end{aligned}
$$


and $T=\eta_{\mu \nu} T^{\mu \nu}$ is the scalar stress-energy. In 3 space dimensions Eq. (79) is readily solved to yield the Newtonian potential [87]:

$$
h_{\mu \nu}(x, t)=\frac{4 G^{(3)}}{\mathrm{c}^{2}} \int \mathrm{d}^{3} x x^{\prime} \frac{\tilde{T}_{\mu \nu}\left(x^{\prime}, t^{\prime}\right)-\frac{1}{2} \eta_{\mu \nu} \tilde{T}\left(x^{\prime}, t^{\prime}\right)}{\left|x-x^{\prime}\right|}
$$

where $t^{\prime}=t-\frac{|x-x \prime|}{\mathrm{c}}$. If $T_{00}=\rho \mathrm{c}^{2}$ is the only non-vanishing element ( $\rho$ : mass density), one has $h_{00}=\frac{2}{\mathrm{c}^{2}} \Phi_{\text {Newton }}=\frac{2}{\mathrm{c}^{2}} \frac{G^{(3)} M_{\text {ext }}}{r}[88]$.

\subsection{Emerging Gravonons: the Massive Particles of a Non-relativistic Gravity Field}

For a free gravitational field the Euler-Lagrange equation (79) reduces to

$$
\partial^{2} h_{\mu \nu}=0
$$

As particles of molecular size couple only to short wavelength gravitons (i.e., quantized gravitational waves) we can write the relativistic energy $E$ of the gravitons as

$$
E=k \mathrm{c}+\epsilon
$$

with $k=|\mathbf{k}|$ and $\mathbf{k}$ labelling the Fourier component of $h_{\mu \nu}$. (In this section the velocity of light c will be explicitly displayed.) With $k$ around $10 \mathrm{bohr}^{-1} E$ will be of the order of $10^{3}$ Hartree, whereas $\epsilon$ characterizes the energy scale with which the boson matter field $\phi$ couples to the gravitons and is of the order of $10^{-12}$ Hartree. (We need short wavelength gravitons with $\lambda \approx 1$ bohr for the localization of particles of atomic size.) The energy $E$ and the length $k$ of the wave vectors involved are therefore defined with a relative precision of $10^{-15}$. This motivates us to write for the graviton field:

$$
h_{\mu \nu}(x)=e^{-\mathrm{i} k \mathrm{ct}} \zeta_{\mu \nu}(x)
$$

The gravonons $\zeta_{\mu \nu}$ contain the gravitational potential and are, of course, not simple number operators. Writing Eq. (83) (Klein-Gordon equation for the field $h_{\mu \nu}$ with $m=0)$ as

$$
\left(\frac{\partial^{2}}{\partial t^{2}}-\mathrm{c}^{2} \nabla^{2}\right) h_{\mu \nu}=0
$$

and, plugging in Eq. (85), yields the following relationships:

$$
\frac{\partial}{\partial t} h_{\mu \nu}=(-\mathrm{i} k \mathrm{c}) h_{\mu \nu}+e^{-\mathrm{i} k \mathrm{c} t} \frac{\partial}{\partial t} \zeta_{\mu \nu}
$$


The second derivative is then

$$
\frac{\partial^{2}}{\partial t^{2}} h_{\mu \nu}=(-\mathrm{i} k \mathrm{c}) e^{-\mathrm{i} k \mathrm{c} t}\left[-\mathrm{i} k \mathrm{c} \zeta_{\mu \nu}+2 \frac{\partial}{\partial t} \zeta_{\mu \nu}+\frac{1}{(-\mathrm{i} k \mathrm{c})} \frac{\partial^{2}}{\partial t^{2}} \zeta_{\mu \nu}\right]
$$

With $\frac{\partial^{2}}{\partial t^{2}} \zeta_{\mu \nu}$ being of order $\epsilon^{2} \approx 10^{-24}$ Hartree $^{2}$ this term can safely be omitted and we obtain for Eq. (86):

$$
-\mathrm{i} k \mathrm{c} \zeta_{\mu \nu}+2 \frac{\partial}{\partial t} \zeta_{\mu \nu}=-\frac{\mathrm{c}}{\mathrm{i} k} \nabla^{2} \zeta_{\mu \nu}
$$

Multiplying by $\frac{\mathrm{i}}{2}$ yields the Schrödinger equation:

$$
\mathrm{i} \frac{\partial}{\partial t} \zeta_{\mu \nu}=\left(-\frac{\nabla^{2}}{2 m_{g}}+V_{o}\right) \zeta_{\mu \nu}
$$

with $m_{g}=\frac{k}{\mathrm{c}}$ the mass of the emerging gravonon and $V_{o}=-\frac{k \mathrm{c}}{2}$ a constant potential cancelling the zero point energy of the light cone graviton $h_{\mu \nu}(\mathbf{k})$. With $k=10 \mathrm{bohr}^{-1}$ the mass $m_{g}$ of the gravonon is roughly a tenth of the mass of an electron.

\subsubsection{The Free Gravonon Action}

In this section the action of the free and the perturbed gravonon field via $\delta S_{\text {matter }}$ is derived. The Schrödinger equation (90) can be obtained by varying the following non-relativistic action with respect to $\zeta_{\mu \nu}^{+}$:

$$
S_{\text {gravonon }}=\int \mathrm{d} t L_{\text {gravonon }}=\int \mathrm{d} t \mathrm{~d}^{D} x\left[\mathrm{i} \zeta_{\mu \nu}^{+} \frac{\partial}{\partial t} \zeta_{\mu \nu}+\frac{1}{2 m_{g}} \zeta_{\mu \nu}^{+} \nabla^{2} \zeta_{\mu \nu}-\zeta_{\mu \nu}^{+} V_{o} \zeta_{\mu \nu}\right]
$$

in $D$ spacial dimensions. This is, however, not the action obtained by inserting Eq. (85) in $S_{w f g}-\delta S_{\text {matter }}$ (cf. Eq. 77). One has:

$$
2 m_{g} S_{\text {gravonon }}=S_{w f g}-\delta S_{\text {matter }}
$$

Determining $\zeta_{\mu \nu}^{+}, \zeta_{\mu \nu}$ from Eq. (91) yields results for $\sqrt{2 m_{g}} \zeta_{\mu \nu}$. Therefore such a solution has to be divided by $\sqrt{2 m_{g}}$ before plugging into $T_{00}$.

Potential terms can be constructed from Eq. (75) together with Eq. (95) yielding expressions like $\zeta_{\mu \nu} L_{\text {matter }}$, which contain terms linear and quadratic in $\zeta_{\mu \nu}$. The linear terms will then be taken into account by shifting the gravonon operator whereas the quadratic terms are inserted into Eq. (90) before solving for $\zeta_{\mu \nu}$. 


\subsection{Matter Fields}

A massive scalar boson field $\phi$ representing the matter fields is introduced as [63]

$$
S_{\text {matter }}=-\int \mathrm{d}^{4} x \sqrt{-g}\left(\frac{1}{2} g^{\mu \nu} \partial_{\mu} \phi^{+} \partial_{\nu} \phi-\frac{1}{2} m^{2} \phi^{+} \phi\right) .
$$

$\phi$ lives only in four dimensional spacetime. It is extended to $N$ dimensional spacetime by setting all values of $\phi(x)$ having $x^{\lambda}>0$ for $\lambda>3$ to zero.

The stress-energy tensor is obtained from Eq. (64) by varying the action of the matter fields with respect to the metric:

$$
T_{\mu \nu}=\partial_{\mu} \phi^{+} \partial_{\nu} \phi-g_{\mu \nu}\left(\frac{1}{2} g^{\rho \lambda} \partial_{\rho} \phi^{+} \partial_{\lambda} \phi-\frac{1}{2} m^{2} \phi^{+} \phi\right) \text {. }
$$

This is a textbook result and it is valid in general before linearization. Before plugging this into Eq. (79) we linearize the stress-energy tensor by utilizing Eq. (65) in the following way:

$$
T_{\mu \nu}=\partial_{\mu} \phi^{+} \partial_{\nu} \phi-\left(\eta_{\mu \nu}+h_{\mu \nu}\right)\left[\frac{1}{2}\left(\eta^{\rho \lambda}+h^{\rho \lambda}\right) \partial_{\rho} \phi^{+} \partial_{\lambda} \phi-\frac{1}{2} m^{2} \phi^{+} \phi\right] \text {. }
$$

For operations on the field $\phi$ the indices run from zero to 3 , for $\eta$ and $h$ they run from 0 to $N$. In this linearized form the stress-energy tensor retains contributions from the gravitational field $h_{\mu \nu}$ and the interaction term Eq. (75) of the action contains terms quadratic in the gravitational field $h_{\mu \nu}$. This kind of self-interaction of the gravitational field turns out to be essential for understanding adsorbate diffusion on solid surfaces and telegraph like quantum jumps (cf. the analysis in Ref. [69] and Sect. 5.1.)

\section{Non-relativistic Limit of the Massive Scalar Boson Field}

The following argument is taken from Ref. [63]. The Klein-Gordon equation for a free scalar boson field of mass $m$ is:

$$
\left(\partial^{2}+m^{2}\right) \phi=0
$$

For a non-relativistic particle (in the sense of special relativity) the kinetic energy is much smaller than its mass $m$ (the velocity of light $\mathrm{c}$ is unity in our notation, as well as $\hbar)$ and the relativistic energy $E$ is written as

$$
E=m+\varepsilon
$$

A boson field $\psi$ varying slowly in time can then be defined:

$$
\phi(x)=e^{-\mathrm{i} m t} \psi(x) .
$$


Setting

$$
\frac{\partial^{2}}{\partial t^{2}} \psi=0
$$

Schrödinger's equation is obtained:

$$
\mathrm{i} \frac{\partial}{\partial t} \psi=-\frac{\nabla^{2}}{2 m} \psi
$$

with $\nabla=\left(\frac{\partial}{\partial x^{1}}, \frac{\partial}{\partial x^{2}}, \ldots, \frac{\partial}{\partial x^{D}}\right)$ for $D$ spacial dimensions. For more details see Ref. [63].

As the matter field $\psi$ lives only in four dimensional spacetime, the Schrödinger equation can be obtained by varying the following non-relativistic action with respect to $\psi^{+}$:

$$
\begin{aligned}
S_{\text {matter-nonrel }} & =\int \mathrm{d} t \mathrm{~d}^{3} x \mathcal{L}_{\text {matter-nonrel }}=\int \mathrm{d} t L_{\text {matter-nonrel }} \\
& =\int \mathrm{d} t \mathrm{~d}^{3} x\left[\mathrm{i} \psi+\frac{\partial}{\partial t} \psi+\frac{1}{2 m} \psi^{+} \nabla^{2} \psi\right]
\end{aligned}
$$

This form of the action linear in $\frac{\partial}{\partial t}$ is obtained by partial integration (cf. Ref. [63]). This is, however, not the action obtained by inserting Eq. (98) in $S_{\text {matter }}\left(h_{\mu \nu}=0\right)$ (Eq. 93). One has:

$$
2 m S_{\text {matter-nonrel }}=S_{\text {matter }}\left(h_{\mu \nu}=0\right) .
$$

The variation of the action Eq. (101) yields a solution for $\sqrt{2 m} \psi$. The solution obtained in this way must hence be divided by $\sqrt{2 m}$ before plugging into $T_{00}$.

\subsection{The Matter-Gravonon Coupling}

The Lagrangian of the matter-gravonon interaction is the focus in the present section. In the non-relativistic limit only $h_{00}$ and $T_{00}$ need to be considered, all other components being smaller by a factor $\left(\frac{v}{c}\right)^{2}$ where $v$ is the non-relativistic velocity. Plugging Eqs. (98) and (85) into Eq. (95) yields $\left(\eta_{00}=1\right)$ :

$$
\begin{aligned}
T_{00}= & \partial_{0} \phi^{+} \partial_{0} \phi-\left(\frac{1}{2} \partial_{0} \phi^{+} \partial_{0} \phi-\frac{1}{2} m^{2} \phi^{+} \phi\right) \\
& -h_{00}\left(\frac{1}{2} \partial_{0} \phi^{+} \partial_{0} \phi-\frac{1}{2} m^{2} \phi^{+} \phi\right)-\frac{1}{2} h^{00} \partial_{0} \phi^{+} \partial_{0} \phi \\
= & \partial_{0} \phi^{+} \partial_{0} \phi-\left(\frac{1}{2} \partial_{0} \phi^{+} \partial_{0} \phi-\frac{1}{2} m^{2} \phi^{+} \phi\right)+h_{00} \frac{1}{2} m^{2} \phi^{+} \phi \\
= & \frac{1}{2} \partial_{0} \phi^{+} \partial_{0} \phi+\frac{1}{2} m^{2} \phi^{+} \phi+h_{00} \frac{1}{2} m^{2} \phi^{+} \phi
\end{aligned}
$$


(We use $h_{00}=-h^{00} ; \psi \partial_{0} \psi^{+}=\psi^{+} \partial_{0} \psi ; \phi(x)=e^{-\mathrm{i} m t} \psi(x) ; \phi^{+}(x)=e^{\mathrm{i} m t} \psi^{+}(x)$; $h_{00}=e^{-\mathrm{i} k \mathrm{ct}} \zeta_{00}$.) Lines 1 and 2 are the standard textbook result. Line 3 contains terms arising from $h_{\mu \nu}$ in Eq. (95). From line 3 to line 4 we used $h^{00}=-h_{00}$ which follows from

$$
\begin{aligned}
\left(1+h_{00}\right)^{-1} & =1+h^{00} \\
& \approx 1-h_{00} .
\end{aligned}
$$

Up to here the expression is relativistically invariant. We now insert the definition Eq. (98).

$$
\begin{aligned}
T_{00}= & \frac{1}{2}\left[m^{2} \psi^{+} \psi+\partial_{0} \psi^{+} \partial_{0} \psi+\mathrm{i} m \psi^{+} \partial_{0} \psi-\mathrm{i} m \psi \partial_{0} \psi^{+}\right] \\
& +\frac{1}{2} m^{2} \phi^{+} \phi+\frac{1}{2} e^{-\mathrm{i} k \mathrm{ct}} \zeta_{00} m^{2} \phi^{+} \phi \\
= & m^{2} \psi^{+} \psi+\frac{1}{2} \partial_{0} \psi^{+} \partial_{0} \psi+\frac{1}{2} e^{-\mathrm{i} k \mathrm{c} t} \zeta_{00} m^{2} \psi^{+} \psi \\
\approx & m^{2} \psi^{+} \psi\left(1+\frac{1}{2} e^{-\mathrm{i} k \mathrm{c} t} \zeta_{00}\right) .
\end{aligned}
$$

In the first two lines of Eq. (105) we used Eq. (85). From lines 1 and 2 to line 3 we used the ansatz Eq. (98). In the last line we used that $\psi$ is slowly varying in time and hence $\frac{1}{2} \partial_{0} \psi^{+} \partial_{0} \psi$ is much smaller than $m^{2} \psi^{+} \psi$.

Now in the lowest order, the Newtonian limit, the expectation value of $T_{00}$ has to be

$$
\left\langle\Psi\left|T_{00}\right| \Psi\right\rangle=\rho \mathrm{c}^{2}=m \mathrm{c}^{2}\left\langle\psi^{+} \psi\right\rangle
$$

with $|\Psi\rangle$ the world wave function. However, using Eq. (105),

$$
\left\langle\Psi\left|T_{00}\right| \Psi\right\rangle=\left\langle\Psi\left|m^{2} \psi^{+} \psi\right| \Psi\right\rangle=m^{2}\left\langle\Psi\left|\psi^{+} \psi\right| \Psi\right\rangle \text {. }
$$

This can only be achieved with

$$
\psi \rightarrow \sqrt{\frac{1}{m}} \psi
$$

If $\psi$ in Eq. (105) is obtained from Eq. (101), then $\psi$ is too large by a factor of $\sqrt{2 m}$ and Eq. (105) has to be divided by $2 m$. If $\zeta_{00}$ in Eq. (105) is obtained from Eq. (91), then $\zeta_{00}$ is too large by a factor of $\sqrt{2 m_{g}}$ and $\zeta_{00}^{+} \zeta_{00}$ in Eq. (105) has to be divided by $2 m_{g}$.

The interaction Lagrangian (matter field with gravitational field), i.e., the integrand in Eq. (75), using Eq. (105), is then:

$$
\frac{1}{2} h_{00} T^{00}\left(1-\frac{1}{2} h^{00}\right)=\frac{1}{2} h^{00} T_{00}\left(1-\frac{1}{2} h^{00}\right)
$$




$$
\begin{aligned}
& =\frac{1}{2} e^{\mathrm{i} k \mathrm{ct}} \zeta_{00}^{+} T_{00}\left(1-\frac{1}{2} h^{00}\right) \\
& \approx \frac{1}{2} m^{2} \psi^{+} \psi\left(e^{\mathrm{i} k \mathrm{c} t} \zeta_{00}^{+}+\frac{1}{2} \zeta_{00}^{+} \zeta_{00}\right)\left(1-\frac{1}{2} h^{00}\right) \\
& =\frac{1}{2} m^{2} \psi^{+} \psi\left(h_{00}+\frac{1}{2} \zeta_{00}^{+} \zeta_{00}-\frac{1}{2} h_{00} h^{00}-\frac{1}{4} \zeta_{00}^{+} \zeta_{00} h^{00}\right)
\end{aligned}
$$

where Eq. (85) has been used in the second line, $h_{00}$ is real:

$$
h_{00}=h_{00}^{*}=-h^{00}=-h^{00 *}=e^{-\mathrm{i} k \mathrm{c} t} \zeta_{00}=e^{\mathrm{i} k \mathrm{c} t} \zeta_{00}^{+}
$$

implying $h_{00} T^{00}=h^{00} T_{00}$ and $h_{00} h^{00}=\zeta_{00}^{+} \zeta_{00}$. In 4 dimensional spacetime $h_{00}$ is proportional to the Newtonian potential obtained from Eq. (82) and according to Ref. [88] should be equal to $h_{00}=\frac{2}{\mathrm{c}^{2}} \Phi_{\text {Newton }}=\frac{2}{\mathrm{c}^{2}} \frac{G^{(3)} M_{\text {ext }}}{r}$. Observe that there is no term linear in $\zeta_{00}$ in Eq. (108), i.e., there is no dipole interaction between the matter field and the gravonons. The terms involving $\zeta_{00}^{+} \zeta_{00}$ (arising from terms $h^{00} h^{00}$ and $\zeta_{00}^{+} h^{00}$ ) signify gravonon-gravonon interaction. They contain the square of the gravitational potential.

The Lagrangian describing the interaction of the scalar boson with Newton's potential and the gravonon field is then (omitting the higher order term proportional to $h_{00} h^{00}$ and setting $h_{00}=\frac{2}{\mathrm{c}^{2}} \frac{G^{(D)} M_{e x t}}{r^{D-2}}$ and $\left.\mathrm{c}=1\right)$ :

$$
\begin{aligned}
L_{i n t} & =\int \mathrm{d}^{D} x \mathcal{L}_{i n t} \\
& =\int \mathrm{d}^{D} x\left[\frac{G^{(D)} m M_{\text {ext }}}{r^{D-2}} \psi^{+} \psi+\frac{m}{2} \zeta_{00}^{+} \zeta_{00} \psi^{+} \psi-\frac{1}{4} \frac{G^{(D)} m M_{\text {ext }}}{r^{D-2}} \zeta_{00}^{+} \zeta_{00} \psi^{+} \psi\right] .
\end{aligned}
$$

$D$ is the number of spacial dimensions. The first term in the second line might be termed the Schrödinger-Newton contribution as in four dimensional spacetime it is the interaction term in the Schrödinger-Newton equation [1]. The second term is the scalar boson-gravonon interaction. The third term describes the interaction between the Schrödinger-Newton term and the gravonons and renormalizes Schrödinger-Newton term:

$$
L_{i n t}=\int \mathrm{d}^{D} x\left[\frac{G^{(D)} m M_{\text {ext }}}{r^{D-2}}\left(1-\frac{1}{4} \zeta_{00}^{+} \zeta_{00}\right) \psi^{+} \psi+\frac{m}{2} \zeta_{00}^{+} \zeta_{00} \psi^{+} \psi\right]
$$

\subsection{Effective Schrödinger Equations}

Adding $\mathcal{L}_{\text {int }}$ (Eq. 111) to the Lagrangian density $\mathcal{L}_{\text {matter-nonrel }}$ of Eq. (101) and varying with respect to $\psi^{+}$yields the Schrödinger equation 


$$
\mathrm{i} \frac{\partial}{\partial t} \psi=\left[-\frac{\nabla^{2}}{2 m}-\frac{G^{(D)} m M_{\text {ext }}}{r^{D-2}}\left(1-\frac{1}{4} \zeta_{00}^{+} \zeta_{00}\right)-\frac{m}{2} \zeta_{00}^{+} \zeta_{00}\right] \psi
$$

which we have to solve simultaneously with the Schrödinger equation arising from varying $\mathcal{L}_{\text {gravonon }}\left(\right.$ Eq. 91 ) and $\mathcal{L}_{\text {int }}$ of Eq. (111) with respect to $\zeta_{00}^{+}$

$$
\mathrm{i} \frac{\partial}{\partial t} \zeta_{00}=\left[-\frac{\nabla^{2}}{2 m_{g}}-\frac{m}{2} \psi^{+} \psi+\frac{1}{4} \frac{G^{(D)} m M_{\text {ext }}}{r^{D-2}} \psi^{+} \psi\right] \zeta_{00}+\left(V_{o}-\frac{k c}{2} h_{00}\right) \zeta_{00}
$$

\subsection{Effective Lagrangian}

The effective Lagrangian is now constructed by adding the Lagrangians contained in the actions of Eqs. (91), (101) and (111)

$$
L_{\text {eff }}=L_{\text {matter-nonrel }}+L_{\text {gravonon }}+L_{\text {int }} .
$$

From this the effective Hamiltonian is deduced with the help of the familiar relationship

$$
H_{e f f}=\int \mathrm{d}^{D} x \frac{\partial L_{\text {matter-nonrel }}}{\partial\left(\partial_{t} \psi\right)} \partial_{t} \psi+\int \mathrm{d}^{D} x \frac{\partial L_{\text {gravonon }}}{\partial\left(\partial_{t} \zeta_{00}\right)} \partial_{t} \zeta_{00}-L_{e f f},
$$

yielding:

$$
\begin{aligned}
H_{\text {eff }} & =H_{\text {gravonon }}^{o}+H_{\text {matter }}^{o}-L_{\text {int }} \\
& =\int \mathrm{d}^{D} x\left[-\psi^{+} \frac{\nabla^{2}}{2 m} \psi-\zeta_{00}^{+} \frac{\nabla^{2}}{2 m_{g}} \zeta_{00}+\left(V_{o}-\frac{k \mathrm{c}}{2} h_{00}\right) \zeta_{00}^{+} \zeta_{00}\right]-L_{\text {int }} .
\end{aligned}
$$

This is the quantum field theoretical Hamiltonian which we have to solve, as it is described in Sect. 2.2, for the problems of quantum particles localization, the diffusion problem, wave-to-particle transition, etc.

\section{Model for the Non-interacting and the Matter-induced Gravonon Structure}

The gravonon field in the second and third terms of the effective Hamiltonian Eq. (116) is a free field in a constant potential. The adsorbate motion, which we want to investigate, occurs, however, in the presence of other masses, which have already induced a structure in the gravonon field. The modelling of the pre-existing gravonon structure has been described in a previous paper [70].

The modification of the gravonon structure induced by matter fields has also been described in Ref. [70] and refers to the generation of soft gravonons. The soft gravonon 
mode is an adsorbate induced coherent motion within a spacetime deformation involving 5 to 10 atoms in the solid surface. The local intrinsic gravonon coherence is retained unless it is destroyed by other fields in the environment.

In Eq. (116) we develop the gravonon fields as follows:

$$
\begin{aligned}
& \zeta_{00}=\sum_{i} b_{i} \zeta_{00}^{(i)} V_{\text {grav }}\left(x_{i}\right) \theta \\
& \zeta_{00}^{+}=\sum_{i} b_{i}^{+} \zeta_{00}^{*(i)} V_{\text {grav }}\left(x_{i}\right) \theta
\end{aligned}
$$

$\zeta_{00}^{(i)}, \zeta_{00}^{*(i)}$ are functions localized near the positions $x_{i}$ of the atomic cores. The gravitational potential $V_{\text {grav }}\left(x_{i}\right)$ has been extracted from the localized fields. The factor $\theta$ serves to make the expansion coefficients $b_{i}, b_{i}^{+}$dimensionless. The effective Hamiltonian Eq. (116) provides for the term $H_{\text {gravonon }}^{o}$ :

$$
\begin{aligned}
H_{\text {gravonon }}^{o} & =\int \mathrm{d}^{D} x \zeta_{00}^{+}\left(-\frac{\nabla^{2}}{2 m_{g}}+V_{o}-\frac{k \mathrm{c}}{2} h_{00}\right) \zeta_{00} \\
& =\sum_{i, j}\left\langle\zeta_{00}^{*(i)}\left|V_{\text {grav }}\left(x_{i}\right)\left(-\frac{\nabla^{2}}{2 m_{g}}+V_{o}-\frac{k \mathrm{c}}{2} h_{00}\right) V_{\text {grav }}\left(x_{j}\right)\right| \zeta_{00}^{(j)}\right\rangle \theta^{2} b_{i}^{+} b_{j} \\
& =\sum_{i, j} \Omega_{i j} b_{i}^{+} b_{j}
\end{aligned}
$$

where $\langle\cdots\rangle$ indicates the volume integration in the $D$-dimensional space and $\Omega_{i j}$ is defined by comparing the last two lines. This is a mode expansion as it is common in quantum field theory. $b_{i}, b_{j}^{+}$are boson operators obeying the commutation relations $\left[b_{i}, b_{j}^{+}\right]=\delta_{i j} . \theta$ is such that $\Omega_{i j}$ has dimension of energy.

If we diagonalize at this place, we obtain a collection of independent harmonic oscillators:

$$
H_{\text {gravonon }}^{o}=\sum_{k} \Omega_{k} b_{k}^{+} b_{k} .
$$

The $\Omega_{k}$ have to be modelled according to the physical situation under investigation. For this purpose we write the boson operators $b_{k}, b_{k}^{+}$in terms of generalized coordinates and momenta:

$$
\begin{aligned}
b_{k}^{+} & =\left(\frac{-\mathrm{i}}{\sqrt{2 \omega_{k}}} p_{k}+\sqrt{\frac{\omega_{k}}{2}} q_{k}\right) \\
b_{k} & =\left(\frac{\mathrm{i}}{\sqrt{2 \omega_{k}}} p_{k}+\sqrt{\frac{\omega_{k}}{2}} q_{k}\right) .
\end{aligned}
$$

The diagonal form Eq. (120) is in the present context considered to represent the eigenmodes of oscillating spacetime deformations centered around atomic cores coupled 
to each other by terms quadratic in the displacements of the cores. In this case we have from Eqs. (117) and (121):

$$
\zeta_{00}^{+}(x)+\zeta_{00}(x)=\sum_{i} 2 q_{i} g\left(x-x_{i}\right)
$$

with

$$
g\left(x-x_{i}\right)=\sqrt{\frac{\omega_{i}}{2}} \zeta_{00}^{i} V_{g r a v}\left(x_{i}\right) \theta
$$

where we now assume that $\zeta_{00}^{i}$ is real. These last two equations should replace Eqs. (12) and (13) in Ref. [70]. For the "potential term" in $\zeta_{00}^{+}(x) \zeta_{00}(x)$ we have then from Eqs. (117) and (121):

$$
\zeta_{00}^{+}(x) \zeta_{00}(x)=\cdots+\sum_{i, j} q_{i} q_{j} g\left(x-x_{i}\right) g\left(x-x_{j}\right) .
$$

This equation should replace Eq. (14) in Ref. [70]. In order to describe effects due to mass motion one has to add terms to Eq. (124) which are not solely a function of $x-x_{i}$ (many-body forces). This has been described in Ref. [70].

\subsection{Induced Scattering in the Gravonon Continuum Leads to Quantum Jumps}

The equations derived in Sects. 4 and 5 have been applied to problems of adsorbate localization [69], adsorbate diffusion [70], scattering of massive particles from nano lattices [74], behaviour of quantum computers [50]. In the case of adsorbate diffusion a discrepancy of nearly nine orders of magnitude between the results of CQM and experiment could be removed. Our theory allows to determine the adsorbate-surface distances from the experimental diffusion rates which are then found to be in good agreement with distances determined from ab-initio density functional calculations [70]. In these model studies quantum jumps arise as sudden changes of the weights of two or more configurations with time. In CQM collapses of the wave function are postulated to result in quantum jumps, whereas in the present EQM they appear as solutions of Schödinger's equation within a unitary coherent quantum description [71]. In the limit of a very local and weak interaction with the gravonons the dynamics of an adsorbed atom between two adsorption sites $\alpha$ and $\beta$ on a solid surface was described by the time development of the occupation of the sites. The occupation of each site was expressed as the sum of the weights of those configurations which involve site $\alpha$ or $\beta$ site, respectively. The time variation of these configurations exhibits the telegraph character and warrants that the occupation of each site jumps between two values (cf. Fig. 1).

\section{Interpretation and Ontology of Emerging QM}

Emerging QM emerges from a quantum field theory which is Lorentz invariant: 
- The world wave functional is a result of the entanglement between local massive particles and the gravonon field in hidden spacial dimensions. The wave functional in Emerging QM does not have the meaning of a probability amplitude. No density matrix and tracing out are needed or involved.

- Massive non-relativistic locally modified soft modes named gravonons are generated in all spacetime dimensions, including the hidden dimensions, due to local gravitational interactions. They modify the time development of the local system in the real 3+1 dimensional world. Beables constitute the connection to experience.

- Observables are not needed since measurement is treated as a quantum process.

- Quantum jumps are intrinsic to the wave functional of Emerging QM and they result from the solution of the time dependent Schrödinger equation in high dimensional spacetime. They are due to the entanglement of the local system to gravonons of high density of states which propagate in hidden spacial dimensions.

- How do we come to the real 3+1 dimensional world? In all experiments discussed in the introduction we see effects which occur locally: either probe particles in the experimental source (electrons, photons, adsorbed particles) or electrons and photons on oscillograph screens and photographic plates, adsorbed particles localized on definite adsorption sites, etc. We do not see these particles in their flight from the source to the target. And if we would try to see them (to "measure" them) on their way to the target the result of the final measurement would be different. Hence we may conclude that what we see occurs locally and then it is in the $3+1$ dimensional world. The transition from the high-dimension configuration space to the $3+1$ dimensional real world in Emerging QM occurs because of the localization of particles via entanglement to gravonons. Just those local components of the wave functional defined in high-dimensional configuration space, which are entangled with the gravonons, define the beables and represent real particles in the $3+1$ real world.

- "Measurement" in Emerging QM is the occurrence of entanglement of a local system with the gravonons. Different outcomes are the result of different initial states. Apparent statistics in the outcome is the consequence of different initial states and hence different experimental conditions.

- Nonlocality is in the high-dimensional wave functional defined in configuration space.

- Emerging QM is Lorentz invariant.

- Emerging QM explains: telegraph signals (quantum jumps), low temperature adsorbate diffusion, adsorbate desorption induced by the current in the scanning tunnelling microscope, Penning ionization at surfaces, etc. (cf. the Introduction).

\section{Comparison Between Emerging QM and Copenhagen and Bohmian QM}

The major ontological features of Emerging QM are compared with those of Copenhagen and Bohmian QM $[18,89]$ in Table 1. The key double-slit experiment can be used to illustrate the points of view in the three theories. 
- Copenhagen QM: Wave-particle duality, collapse and probability interpretation are used in a phenomenological way to provide the explanation of how the diffraction pattern is created on the detection screen. Schrödinger's wave function interferes with itself. At different screen positions the "measurement" consists of instantaneous transformation of waves into particles (called collapses) with probabilities, determined by Born's rule, namely by the modulus squared of the amplitude of the wave function at that position. The interference pattern in the wave function is imprinted on the detection screen via probabilistic collapses of the wave function at different positions on the screen at different times.

- Bohm's theory: The wave function due to standard quantum mechanics develops the interference pattern when it interferes with itself. Single particle trajectories pass through one slit only, however the interference pattern, which develops in the wave function, guides the particles in regions of constructive interference, avoiding regions of destructive interference. The final position of a particle on the screen and which slit it will choose to pass through is determined by the initial position of the particle (which is not controlled by the experimenter) and by the time development of the wave function. In the $3+1$ real world this is expressed by the existence of regions where particle trajectories are dense and regions with less dense particle trajectories, providing the diffraction pattern on the detection screen.

- Emerging QM describes the double-slit experimental result as due to the world wave functional propagating through both slits and interfering with itself. The interference pattern is built into the wave functional already in the vacuum region between the source, the slits and the detection screen. On the screen, where matter density is high, components of the world wave functional entangle locally with the gravonons, leading to particles localizing on the screen. This occurs more often with components, which have high amplitude in the total wave functional, i.e. particle localization at selected positions on the screen occur more often for repeated experiments, giving rise to the diffraction pattern.

In Table 1 an attempt is made to identify similarities and differences in the ontologies of CQM, Bohm's QM and Emerging QM.

As it is evident from Table 1 there are many features shared by Bohmian QM and Emerging QM. We emphasize two point: (i) Both for Bohmian QM and Emerging QM the measurement problem and collapse do not exist and (ii) Particles in $3+1$ dimensional space exist, i.e. local realism is implicit. In Emerging QM these are the configurations which entangle with the gravonons and where the particles are localized at definite positions at definite time. In Bohmian QM these are particles on definite trajectories.

An important difference is that Bohmian QM is not, whereas Emerging QM is Lorentz invariant, being the non-relativistic limit of Quantum field theory. Superluminal changes of particle position are possible in Emerging QM in the absolute non-relativistic limit of extremely weak interactions between the local system and environmental gravonons of zero frequency which eventually might violate causality. However, in all practical situations a large but finite velocity in the behaviour of the 
Table 1 Comparison between the ontology within Copenhagen QM, Bohm's QM and Emerging QM

\begin{tabular}{llll}
\hline Ontological property & Copenhagen QM & Bohm's QM & Emerging QM \\
\hline Determinism & No & Yes & Yes \\
Chooser & $\begin{array}{c}\text { No (“God plays } \\
\text { dice“) }\end{array}$ & $\begin{array}{c}\text { Yes (particle } \\
\text { positions) }\end{array}$ & Yes (gravonons) \\
Collapse & Yes & No & No \\
Measurement problem & Yes & No & No \\
Probabilistic interpretation & Yes & No & No \\
Statistical distribution in initial state & No & Yes & Yes \\
Hidden physical quantities & No & Yes (positions) & Yes (gravitons in \\
& & & hidden \\
& No & Yes & dimensions) \\
Definite outcome & No & Yes & Yes \\
Pre-existing realism in 3+1 dimensions & No & Yes & Yes \\
Particles in 3+1 dimensions & No & Yes & No \\
Quantum equilibrium & No, needs & No & Yes \\
Wave function is complete description & probabilistic & & \\
& interpret & & Yes (it is the limit of \\
Lorentz invariance & No & No & invariant theory) \\
& & & Yes \\
New results compared to Copenhagen QM & & No &
\end{tabular}

local system is obtained in the $3+1$ world as solution of the time dependent Schrödinger equation.

A noteworthy difference with CQM is that, whereas Emerging QM is falsifiable, Copenhagen QM and in particular the notion of random collapse phenomena it assumes, is not.

Emerging QM provides the description of many other experiments which is not possible for Bohmian QM. To mention some of them:

- Adsorbate localization and slow down of diffusion on solid surfaces.

- Telegraph like quantum jumps of adsorbates between adsorption sites in the regime of quantum diffusion at low temperatire, resulting from the time dependent Schrödinger equation.

\section{Conclusion}

Emerging QM, the focus of the present article, is derived from Quantum field theory in the non-relativistic limit. It complies therefore with Lorentz invariance and does not suffer from this deficit in Copenhagen QM and Bohmian QM. Quantum jumps in Emerging QM do not violate causality. 
The theory accounts for nonlocal correlations as a result of local entanglement of particles with the high-dimensional gravitational field. Weak and local interaction with the gravitational field of high density of states leads to particle localization and local realism. Starting from high-dimensional configuration space, we can go to the $3+1$ spacetime as a result of particle localization via entanglement with the gravitational field and explain experimental observations in the real 3+1 dimensional world, not violating Lorentz invariance.

Emerging QM is realistic in a sense similar to Bohm's quantum mechanics. At the same time it is capable of reproducing and explaining experimental results which are outside the scope of Copenhagen QM and Bohmian QM. Within Emerging QM particle localization, adsorbate diffusion on solid surfaces and quantum jumps, as they are experimentally measured in the real $3+1$ world, are the result of the solution of Schrödinger's time dependent equation for a local system which entangles with the high density of states of the gravitational field.

A message of the present contribution is that there is no probabilistic element in the time development resulting from the time dependent Schrödinger equation. The time development of a local quantum system entangled to quantum fields in its environment of high dimensions and high density of states, though it resembles telegraph signal-like quantum jumps, is deterministic. The conditions for the quantum jumps are: weak and local interaction with the environmental degrees of freedom (the gravitational field) with high density of states. Statistical appearance of experiments arises due to different initial states which are not controlled in experiment.

The connection from the high-dimensional configuration space to the real $3+1$ world arises because coupling to the gravitational field occurs only for matter fields localized in three dimensional space and this reproduces the results of experiments in the real world without the need of collapse and state reduction, just as a result of the time development of a local system entangled with the gravitational field continuum, as Schrödinger's equation requires. In the extreme non-relativistic limit of vanishingly weak interaction with gravitational field modes of zero frequency the theory may lead to superluminal quantum jumps. But in all practical situations this is not the case. Being derived from quantum field theory, Emerging QM is Lorentz invariant and is not in conflict with the concept of causality.

The derivation of the equations of Emerging QM and their application to reproduce and understand concrete experiments leads to the conclusion that Schrödinger quantum mechanics has emerged. Furthermore Emerging QM allows the interpretation of quantum mechanics in the real 3+1 world without the concepts of collapse and Born's probability. The crucial feature of Emerging QM is the localization of quantum particles due to entanglement with the modes of the gravitational field in high-dimensional spacetime.

Conflict of interest The authors declare that they have no conflict of interest.

Open Access This article is distributed under the terms of the Creative Commons Attribution 4.0 International License (http://creativecommons.org/licenses/by/4.0/), which permits unrestricted use, distribution, and reproduction in any medium, provided you give appropriate credit to the original author(s) and the source, provide a link to the Creative Commons license, and indicate if changes were made. 


\section{References}

1. Penrose, R.: On gravity's role in quantum state reduction. Gen. Relativ. Gravit. 28, 581-600 (1996)

2. Penrose, R.: Quantum computation, entanglement and state reduction. Philos. Trans. R. Soc. A 356, 1927-1939 (1998)

3. Penrose, R.: The Large, the Small and the Human Mind. Cambridge University Press, New York (1997)

4. Penrose, R.: The Emperor's New Mind. Oxford University Press, Oxford (1992)

5. Penrose, R.: Shadow of the Mind. Oxford University Press, Oxford (1994)

6. Penrose, R.: The Road to Reality-A Complete Guide to the Laws of the Universe. Knopf, New York (2005)

7. Omnès, R.: Are there unresolved problems in the interpretation of quantum mechanics? In: Breuer, H.-P., Petruccione, F. (eds.) Open Systems and Measurement in Relativistic Quantum Theory, pp. 169-194. Springer, Berlin (1999)

8. Bassi, A., Ghirardi, G.C.: Dynamical reduction models. Phys. Rep. 379, 257-426 (2003)

9. Pearle, P.: Collapse models. In: Breuer, H.-P., Petruccione, F. (eds.) Open Systems and Measurement in Relativistic Quantum Theory, pp. 195-234. Springer, Berlin (1999)

10. Begingham, D., Dürr, D., Ghirardi, G.C., Goldstein, S., Tumulka, R., Zanghi, N.: Matter density and relativistic models of wave function collapse. J. Stat. Phys. 154, 623-631 (2014)

11. Tumulka, R.: A relativistic version of the Ghirardi-Rimini-Weber model. J. Stat. Phys. 125, 821-840 (2006)

12. Tumulka, R.: The point processes of the GRW theory of wave function collapse. Rev. Math. Phys. 21, 155-227 (2009)

13. Kiefer, C.: Quantum Gravity. Oxford University Press, Oxford (2007)

14. Kiefer, C.: On the interpretation of quantum theory-from Copenhagen to the present day. In: Castell, L., Ischebeck, O. (eds.) Time, Quantum and Information, pp. 291-292. Springer, Berlin (2003)

15. Kiefer, C., Joos, E.: Decoherence: concepts and examples. In: Blanchard, Ph, Jadczyk, A. (eds.) Quantum Future-From Volta and Como to the Present and Beyond, pp. 105-128. Springer, Berlin (1999)

16. d'Espagnat, B.: Conceptual Foundations of Quantum Mechanics. Benjamin, Menlo Park (1971)

17. d'Espagnat, B.: Problems in objectizing. In: Blanchard, Ph., Jadczyk, A. (eds.) Quantum Future-From Volta and Como to the Present and Beyond, pp. 37-46. Springer, Berlin (1999)

18. Dürr, D., Teufel, S.: Bohmian Mechanics—The Physics and Mathematics of Quantum Theory. Springer, Berlin (2009)

19. von Neumann, J.: Mathematical Foundations of Quantum Mechanics. Princeton University Press, Princeton (1932/1955)

20. Becker, K., Becker, M., Schwarz, J.H.: String Theory and M-Theory. Cambridge University Press, Cambridge (2007)

21. Susskind, L.: The Cosmic Landscape-String Theory and the Illusion of Intelligent Design. Back Bay Books, San Francisco (2006)

22. 't Hooft, G.: Quantum gravity as a dissipative deterministic system. Class. Quantum Gravity 16, 32633279 (1999)

23. 't Hooft, G.: Determinism beneath quantum mechanics.http://arxiv.org/pdf/quant-ph/0212095 (2002)

24. 't Hooft, G.: Determinism in free bosons. Int. J. Theor. Phys. 42, 355-361 (2003)

25. 't Hooft, G.: A mathematical theory for deterministic quantum mechanics. J. Phys. 67, 012015 (2007)

26. Blasone, M., Jizba, P., Vitiello, G.: Dissipation and quantization. Phys. Lett. A 287, 205-210 (2001)

27. Adler, S.L.: Quantum Theory as a Emergent Phenomenon-The Statistical Mechanics of Matrix Models as the Precursor of Quantum Field Theory. Cambridge University Press, Cambridge (2004)

28. Adler, S.L.: Quantum Theory as an Emergent Phenomenon: Foundations and Phenomenogy. J. Phys. 361, 012002 (2012)

29. Smolin, L.: Matrix models as non-local hidden variables theories. http://arxiv.org/pdf/hep-th/0201031 (2002)

30. Biró, T.S., Matinyan, S.G., Müller, B.: Chaotic quantization of classical gauge fields. Found. Phys. Lett. 14, 471-485 (2001)

31. Hadley, M.J.: The logic of quantum mechanics derived from classical general relativity. Found. Phys. Lett. 10, 43-60 (1997)

32. Ghirardi, G.C., Rimini, A., Weber, T.: Unified dynamics for microscopic and macroscopic systems. Phys. Rev. D 34, 470-491 (1986) 
33. Haag, R.: Objects, Events and Localization. In: Blanchard, Ph, Jadczyk, A. (eds.) Quantum FutureFrom Volta and Como to the Present and Beyond, pp. 58-79. Springer, Berlin (1999)

34. Eigler, D.M., Schweizer, E.K.: Positioning single atoms with a scanning tunnelling microscope. Nature (London) 344, 524-526 (1990)

35. Lauhon, L.J., Ho, W.: Direct observation of the quantum tunneling of single hydrogen atoms with a scanning tunneling microscope. Phys. Rev. Lett. 85, 4566-4569 (2000); Erratum: Lauhon, L.J., Ho, W.: Phys. Rev. Lett. 89, 079901 (2002)

36. Liljeroth, P., Repp, J., Meyer, G.: Current-induced hydrogen tautomerization and conductance switching of naphthalocyanine molecules. Science 317, 1203-1206 (2007)

37. Lin, T.S., Gomer, R.: Diffusion of ${ }^{1} \mathrm{H}$ and ${ }^{2} \mathrm{H}$ on the Ni(111) and (100) planes. Surf. Sci. 255, 41-60 (1991)

38. Mattsson, Th, Wahnström, G.: Isotope effect in hydrogen surface diffusion. Phys. Rev. B 56, 1494414947 (1997)

39. Bartels, L., Meyer, G., Rieder, K.-H., Velic, D., Knoesel, E., Hotzel, A., Wolf, M., Ertl, G.: Dynamics of electron-induced manipulation of individual CO molecules on Cu(111). Phys. Rev. Lett. 80, 2004-2007 (1998)

40. Drakova, D., Doyen, G.: Reaction threshold and decoherence: current induced desorption of CO on $\mathrm{Cu}(111)$ in STM. J. Phys. 61, 262-268 (2007)

41. Conrad, H., Ertl, G., Küppers, J., Wang, S.W., Gérard, K., Haberland, H.: Penning-ionization electron spectroscopy of chemisorbed CO. Phys. Rev. Lett. 42, 1082-1086 (1979)

42. Conrad, H., Ertl, G., Küppers, J., Sesselmann, W., Haberland, H.: Electron spectroscopy of surfaces by impact of metastable He atoms: CO on Pd(110). Surf. Sci. 121, 161-180 (1982)

43. Conrad, H., Doyen, G., Ertl, G., Küppers, J., Sesselmann, W.: Reflection of metastable He atoms from solid surfaces. Chem. Phys. Lett. 88, 281-285 (1982)

44. Doyen, G.: Model for handling the transmission problem in sticking at cold surfaces. Phys. Rev. B 22, 497-506 (1980)

45. Brooke, J., Bitko, D., Rosenbaum, T.F., Aepli, G.: Quantum annealing of a disordered magnet. Science 284, 779-781 (1999)

46. http://www.dwavesys.com. Accessed 3 June 2015

47. Chiorescu, I., Nakamura, Y., Harmans, C.J.P.M., Mooij, J.E.: Coherent quantum dynamics of a superconducting flux qubit. Science 299, 1869-1871 (2003)

48. Johnson, M.W., et al.: Quantum annealing with manufactured spins. Nature 473, 194-198 (2011)

49. Dickson, N.G., et al.: Thermally assisted quantum annealing of a 16-qubit problem. Nat. Commun. 4, 1903 (2013). doi:10.1038/ncomms2920

50. Drakova, D., Doyen, G.: How can the D-Wave machine exhibit long-time quantum behaviour. In: Presented at DICE (2014, submitted)

51. Diósi, L.: Models for universal reduction of macroscopic quantum fluctuations. Phys. Rev. A 40, 1165-1174 (1989)

52. Diósi, L.: Notes on certain newton gravity mechanisms of wave function localization and decoherence. http://arxiv.org/quant-ph/060711 (2005)

53. Moroz, I.M., Penrose, R., Tod, K.P.: Spherically-symmetric solution of the Schrödinger-Newton equations. Class. Quantum Gravity 15, 2733-2742 (1998)

54. Ghirardi, G.C., Grassi, R., Rimini, A.: Continuous-spontaneous-reduction model involving gravity. Phys. Rev. A 42, 1057-1064 (1990)

55. Percival, I.C.: Atom interferometry, spacetime and reality. Phys. World 10, $43-48$ (1997)

56. Percival, I.C., Strunz, W.T.: Detection of spacetime fluctuation by a model interferometer. Proc. R. Soc. Lond. A 453, 431-446 (1997)

57. Hughston, L.P.: Geometry of stochastic state vector reduction. Proc. R. Soc. Lond. A 452, 953-979 (1996)

58. Pearle, P., Squires, E.: Bound state excitation, nucleon decay experiments and models of wave function collapse. Phys. Rev. Lett. 73, 1-5 (1994)

59. Pearle, P., Squires, E.: Gravity, energy conservation, and parameter values in collapse models. Found. Phys. 26, 291-305 (1996)

60. Anastopoulos, C.: Quantum theory of nonrelativistic particles interacting with gravity. Phys. Rev. D 54, 1600-1605 (1996)

61. Lamine, B., Hervé, R., Lambrecht, A., Reynauld, S.: Ultimate decoherence border for matter-wave interferometry. Phys. Rev. Lett. 96, 050405 (2006) 
62. Breuer, H.-P., Göklü, E., Lämmerzahl, C.: Metric fluctuations and decoherence. Class. Quantum Gravity 26, 105012 (2009)

63. Zee, A.: Quantum Field Theory in a Nutshell. Princeton University Press, Princeton (2003)

64. Gross, F.: Relativistic Quantum Mechanics and Field Theory, p. 453. Wiley, New York (2008)

65. Arkani-Hamed, N., Dimopoulos, S., Dvali, G., Kaloper, N.: Infinitely large new dimensions. Phys. Rev. Lett. 84, 586-589 (2000)

66. Arkani-Hamed, N., Dimopoulos, S., March-Russell, J.: Stabilization of submillimeter dimensions: the new guise of the hierarchy problem. Phys. Rev. D 63, 064020 (2001)

67. Doyen, G., Drakova, D.: Why is more different: the appearance of microscopic classical particles. J. Phys. 306, 012033 (2011)

68. Zwiebach, B.: A First Course in String Theory. Cambridge University Press, Cambridge (2004)

69. Doyen, G., Drakova, D.: Telegraph signals as a solution of the time dependent Schrödinger equation let standard Copenhagen quantum mechanics emerge. J. Phys. 442, 012032 (2013)

70. Drakova, D., Doyen, G.: Low temperature motion of hydrogen on metal surfaces signals breakdown of quantum mechanics in 3+1 dimensions. J. Phys. 442, 012049 (2013)

71. Drakova, D., Doyen, G.: Telegraph signals as a solution of the time dependent Schrödinger equation. http://arxiv.org/quant-ph/pdf/1204.5606 (2012)

72. Lippmann, B.A., Schwinger, J.: Variational Principles for Scattering Processes. I. Phys. Rev. 79, 469480 (1950)

73. Gradshteyn, I.S., Ryzhik, I.M.: Table of Integrals, Series, and Products (Integral 3.354.5), p. 312. Academic Press, New York (1965)

74. Doyen, G., Drakova, D.: Emergence of four dimensional quantum mechanics from a deterministic theory in 11 dimensions. In: Presented at DICE (2014, submitted)

75. Bell, J.: Beables for quantum field theory. In: Bell, J.S. (ed.) Speakable and Unspeakable in Quantum Mechanics, pp. 171-180. Cambridge University Press, Cambridge (1987)

76. Bell, J.: On the Einstein Podolsky Rosen Paradox. Physics 1, 195-200 (1965)

77. Bell, J.: On the problem of hidden variables in quantum mechanics. Rev. Mod. Phys. 38, 447-452 (1966)

78. Maudlin, T.: Quantum Non-locality and Reality-Metaphysical Intimations of Modern Physics, Chapter 10. Wiley-Blackwell, Oxford (2011)

79. Kant, I.: Critique of Pure Reason, 1781, tr. by N. Kemp Smith. St. Martin's Press, New York (1965)

80. Auyang, S.Y.: How is Quantum Field Theory Possible?, p. 71. Oxford University Press, Oxford (1995), see also notes 69 and 120

81. Fine, A.: The Shaky Game-Einstein Realism and the Quantum Theory, 2nd edn. University of Chicago Press, Chicago (1997)

82. Callender, C.: Finding "real" time in quantum mechanics. In: Craig, W.L., Smith, Q. (eds.) Einstein, Relativity and Absolute Simultaneity, pp. 50-72. Routledge, New York (2011)

83. Einstein, A., Rosen, N., Podolsky, B.: Can quantum-mechanical description of physical reality be considered complete? Phys. Rev. 47, 777-780 (1935)

84. Tooley, M.: A defense of absolute simultaneity. In: Craig, W.L., Smith, Q. (eds.) Einstein, Relativity and Absolute Simultaneity, pp. 229-243. Routledge, London (2011)

85. Albert, D.Z.: Quantum Mechanics and Experience, pp. 155-160. Harvard University Press, Cambridge (1992)

86. Misner, ChW, Thorne, K.S., Wheeler, J.A.: Gravitation. W.H. Freeman, San Francisco (1973)

87. Ryder, L.: Introduction to General Relativity. Cambridge University Press, Cambridge (2009)

88. Cheng, T.-P.: Relativity, Gravitation and Cosmology-A basic Introduction, 2nd edn. Oxford Master Series in Physics, Oxford University Press, New York (2010)

89. Bohm, D.: A suggested interpretation of the quantum theory in terms of "Hidden Variables". Phys. Rev. 85, 165-179, 180-193 (1952) 